\title{
Testing Models for the Quasar Big Blue Bump via Color-Color Diagrams
}

\section{Citation}

Siemiginowska, Aneta, Olga Kuhn, Martin Elvis, Fabrizio Fiore, Jonathan McDowell, and Belinda J. Wilkes. 1995. "Testing Models for the Quasar Big Blue Bump via Color-Color Diagrams." The Astrophysical Journal 454 (November): 77. doi:10.1086/176467.

\section{Published Version}

doi:10.1086/176467

\section{Permanent link}

http://nrs.harvard.edu/urn-3:HUL.InstRepos:30212171

\section{Terms of Use}

This article was downloaded from Harvard University's DASH repository, and is made available under the terms and conditions applicable to Other Posted Material, as set forth at http:// nrs.harvard.edu/urn-3:HUL.InstRepos:dash.current.terms-of-use\#LAA

\section{Share Your Story}

The Harvard community has made this article openly available.

Please share how this access benefits you. Submit a story.

\section{Accessibility}




\title{
TESTING MODELS FOR THE QUASAR BIG BLUE BUMP VIA COLOR-COLOR DIAGRAMS
}

\author{
Aneta Siemiginowska, Olga Kuhn, Martin Elvis, Fabrizio Fiore, ${ }^{1}$ \\ JoNATHAN MCDOWELl, AND BELINDA J. WiLkES \\ Harvard-Smithsonian Center for Astrophysics, 60 Garden Street, Cambridge, MA 02138 \\ Received 1994 September 6; accepted 1995 May 31
}

\begin{abstract}
We discuss several models of quasar big blue bump emission in color-color and color-luminosity diagrams. We define several broad passbands: IR $(0.8-1.6 \mu \mathrm{m})$, VIS (4000-8000 $\AA)$, UV (1000-2000 $)$, UV1 (1400-2000 $\AA)$ and UV2 (1000-1400 $\AA$ ), and SX $(0.2-0.4 \mathrm{keV})$. The colors have been chosen to investigate characteristics of the big blue bump: (1) IR/VIS color represents the importance of the IR component and shows the contribution around $\sim 1 \mu \mathrm{m}$; (2) UV/VIS color shows the slope of the big blue bump (in a region where it dominates, a higher value means the bump gets steeper); (3) the combination of IR/VIS/UV colors shows the relative strength of the big blue bump and the IR component; (4) UV1/UV2 color is important as an indicator of a flattening of the spectrum in this region and the presence of the far-UV turnover, (5) UV/SX tests the relationship between the big blue bump and the soft X-ray component. All colors are needed to investigate the range of model parameters.

We describe the colors for several models: accretion disk models in Schwarzschild and Kerr geometries, single-temperature optically thin emission, combination of the main emission model and nonthermal power law or dust, and irradiation of the disk surface. We test models against the sample of 47 low-redshift quasars from Elvis et al. We find that (1) modified blackbody emission from an accretion disk in a Kerr geometry can successfully reproduce both the luminosities and colors of the quasars except for the soft X-ray emission; (2) no additional components (hot dust or power-law) are needed to fit the optical-UV colors when the irradiation of the surface of the disk is included in the model; (3) even modest $(10 \%)$ irradiation of the surface of the disk modifies significantly the optical colors; (4) the simplest, single-temperature, free-free models need either an additional component or a range of temperatures to explain the observations.

Tables of predicted colors for each model family are provided on the AAS CD-ROM. A part of the tables is listed in the Appendix.

Subject headings: accretion, accretion disks - galaxies: photometry - quasars: general radiation mechanisms: thermal
\end{abstract}

\section{INTRODUCTION}

Quasars emit efficiently over a very broad energy range with a significant fraction (Elvis et al. 1994, hereafter Paper I) of power in the infrared to soft X-ray band. The most prominent feature in quasar spectral energy distributions is an excess of power called the big blue bump, which extends from $\sim 1 \mu \mathrm{m}$ into the unobserved far-ultraviolet band. Shields (1979) suggested that this feature, observed in 3 C 273, may be the result of thermal emission from an accretion disk around a supermassive black hole. This was the first attempt at identifying a signature of the primary quasar emission. While the generally accepted source of quasar luminosity is the release of gravitational energy in the vicinity of a supermassive black hole, the problem of how this energy is converted into the radiation, and which parts of the spectrum show the primary emission component is still open. The origin of the big blue bump emission and its connection to the primary power engine is disputed; both disk and free-free emission have evidence in their favor (Czerny 1994; Antonucci 1994).

In the past 15 years a number of efforts have been made to fit the optical-UV data of quasars and so constrain parameters in a variety of models (Malkan 1983; Bechtold et al. 1987; Sun \& Malkan 1989; Sanders et al. 1989; Laor 1990; Bechtold et al.

\footnotetext{
${ }^{1}$ Present address: Osservatorio Astronomico di Roma via dell'Osservatorio 5, Monteporzio-Catone (RM), I00040 Italy.
}

1994; Kuhn et al. 1995). Much of this work focused on the individual objects rather than on examining global properties of the models and data together. While fits to the individual spectra are generally successful, the large number of parameters renders them ineffective in constraining or excluding any of the models. Several combinations of model parameters usually yield equally good fits. Also, the wide variety of observed strengths and shapes of the big blue bump is not easily seen by examining one object at a time.

The method presented in this paper allows examination of the full available set of data and models simultaneously by means of color-color diagrams. These are valuable in gaining insight to the global properties of data and models. By using fully observed continua (Paper I), we are free to choose constant rest-frame colors, different from the standard photometric, $U B V R I$, bands, that pick out various features of the quasar continuum (e.g., the strength of the big blue bump, the slope of the UV continuum, or the connection between UV and the soft-X-rays). We have constructed colors for sets of models to study how changes in either the model parameters or assumptions (e.g., Kerr vs. Schwarzschild disks) affect the continuum shapes. We make comparisons between the data and two competing models for the blue bump: accretion disk emission and thermal bremsstrahlung. We also discuss the effects of adding other components (a power-law or hot dust) and irradiation of the surface of an accretion disk.

In the next section, color-color diagrams are discussed. 
Section 3 describes the dataset. Section 4 discusses models. In $\S 5$ we compare the models and data. Section 6 summarizes the conclusions that can be made from this study.

\section{COLOR-COLOR DIAGRAMS}

Color-color and color-luminosity diagrams have been widely used in studies of populations of stars (Hertzsprung 1905; Russell 1912, 1913), low-mass X-ray binary systems (Van der Klis 1989), stellar clusters (Sandage 1957), and galaxies (Tinsley 1973). Applying this approach to quasars has the problem that their large range of redshifts causes any set of observed colors to span a wide range of emitted wavelengths within a given sample. This makes it hard to plot colors of models on the same diagram as the data. Quasars have been analyzed in luminosity versus spectral index (Wandel \& Petrosian 1988; Wandel 1991; Tripp, Bechtold, \& Green 1994), color-color (Sandage 1973) and color-luminosity diagrams (Caditz 1993). Caditz (1993) used the readily available $U$ and $B$ bands for samples of quasars (Véron-Cetty \& Véron 1989; Boyle et al. 1990) and built color-luminosity plots at two chosen redshifts. He analyzed the colors in the observed frame, transferring models to the correct frequencies. The method is useful, since a large number of objects is accessible from existing catalogs; however, it requires redshift bins to be small, so that there is no major change in the continuum slope within a redshift bin. Furthermore, when using standard filter bands, the inherently uncertain contribution from any broad emission lines in particular bands has to be taken into account, and some redshifts with large line contributions are thus excluded. Finally, since the same part of the intrinsic spectrum cannot be tracked with redshift, it is not possible to investigate the evolution of the quasar population with this method.

Because we begin with completely sampled continua, we are able to use the same rest frame frequencies for all our analyses. The definition of model colors is thus straightforward, as is the interpretation of colors defined at the source; moreover, emission lines can be avoided. The biggest effort was in preparing the data, since we need to transfer all spectra to the correct rest frame frequencies and correct the data for intergalactic reddening and for the contribution from a host galaxy (this is important for low-luminosity objects).

The observed big blue bump covers more than two decades in frequency, $\sim 10^{14.5}-10^{17} \mathrm{~Hz}(1 \mu \mathrm{m}-0.5 \mathrm{keV})$, although there is no clear evidence that the far-UV and soft X-ray emission are parts of the same component (Fiore et al. 1994, 1995). An adequate representation of the big blue bump thus requires the use of well-separated bands, which must also be broad $\left(\Delta \lambda / \lambda_{0} \sim 1\right)$ to obtain good signal-to-noise ratio $(\mathrm{S} / \mathrm{N})$, especially in the UV.

In order to describe the shape of the blue bump component, we define a set of octave wide bands: IR $(0.8-1.6 \mu \mathrm{m})$, VIS (4000-8000 $\AA)$, UV (1000-2000 $\AA)$, UV1 (1400-2000 $\AA)$ and UV2 (1000-1400 $\AA$ ), and SX $(0.2-0.4 \mathrm{keV})$. A color is a ratio of luminosities in two bands.

Figure 1 shows a typical IR-X-ray quasar spectral energy distribution in the rest frame and shows where each color band lies with respect to the big blue bump. From this, it can be seen that each color studies particular features of the quasar continuum:

1. IR/VIS color shows the contribution around $\sim 1 \mu \mathrm{m}$;

2. UV/VIS color shows the slope of the big blue bump: in a region where it dominates, a higher value means the bump becomes steeper.

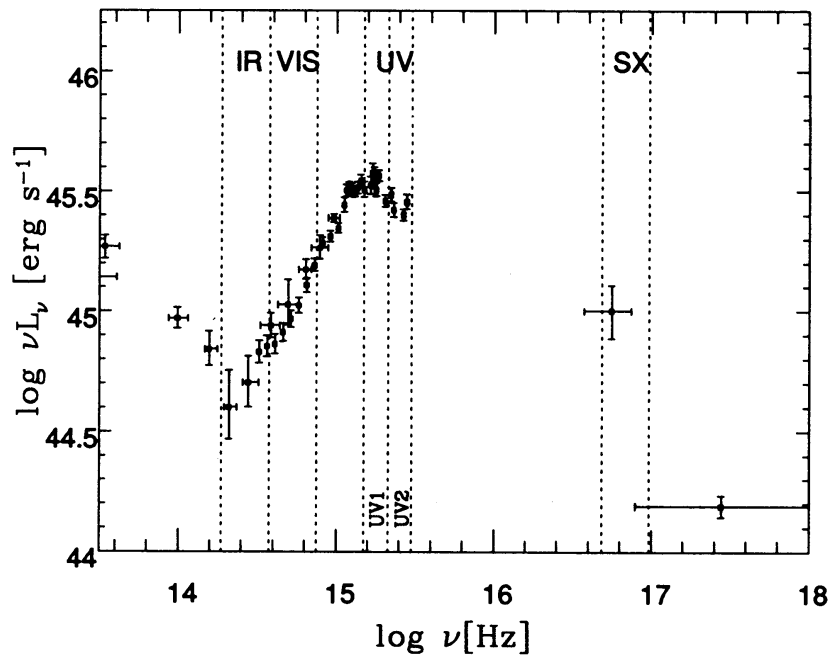

Fig. 1.-Quasar spectral energy distribution of NAB $0205+024$ with indicated luminosity bands.

3. The combination of IR/VIS/UV color shows the relative strength of the big blue bump and the IR component.

4. UV1/UV2 color is important as an indicator of a flattening of the spectrum in this region and the presence of a far-UV turnover. Positive values of $\log (\mathrm{UV} 1 / \mathrm{UV} 2)$ indicate flattening in this band.

5. UV/SX tests the relationship between the big blue bump and the soft $\mathrm{X}$-ray component.

We do not consider the NUV (2000-4000 $\AA$ ) band discussed in Paper I. It covers the near-ultraviolet region of the spectrum dominated by the "small bump" of blended Fe II and Balmer continuum emission (Wills, Netzer, \& Wills 1985).

\section{THE DATA SET}

We considered the sample of 47 quasars (the "UVSX" sample) described in detail in Paper I. All objects in the sample have:

1. UV spectra (1200-3000 $\AA$ ) from $I U E$;

2. Soft X-ray spectra $(0.1-4.0 \mathrm{keV})$ from the Einstein IPC;

3. Spectrophotometry and/or photometry in the optical;

4. Photometric data in the infrared;

5. Radio data.

The quasars are at low redshift $(0.025 \leq z<1$, mostly around $z \sim 0.1)$ and are bright $\left(m_{V}<17\right)$. The bolometric luminosities (integrated from $10^{9}-10^{18} \mathrm{~Hz}$ ) of the sample objects range from about $10^{45}$ ergs $\mathrm{s}^{-1}$ to $10^{47} \mathrm{ergs} \mathrm{s}^{-1}$. We have assumed $H_{0}=50 \mathrm{~km} \mathrm{~s}^{-1} \mathrm{Mpc}^{-1}$ and $\Omega_{0}=1$.

The requirement that the "UV" quasars have more than 300 counts (a signal-to-noise ratio better than $\sim 10$ ) in the IPC (Wilkes \& Elvis 1987) introduces a bias toward objects with a large amount of $\mathrm{X}$-ray emission relative to the optical and also toward those that are relatively optically bright and nearby.

The collection and reduction of these data are discussed in detail in the "Atlas of Quasar Energy Distributions" (Paper I). Briefly, the steps in the reduction of these data were the following:

1. Dereddening by the Galactic value;

2. Blueshifting by $(1+z)$ and binning data into line-free frequency bins;

3. Subtracting emission lines; 
4. Subtracting a template host galaxy;

5. Time averaging the data within the same frequency bin.

We list all quasars in Table 1. The table gives the common name of the quasar and the name of the associated host galaxy where appropriate, the redshift, and typical $V$ magnitude. Each object is given a classification: radio-quiet $(\mathrm{RQ})$ or radio-loud (RL). Following the convention of Véron-Cetty \& Véron (1989) and Schmidt \& Green (1983), radio-quiet objects with abolute visual magnitude fainter than -23.0 calculated according to their prescription (but using our cosmological parameters) are designated as Seyfert 1 (Sy1); eight objects in the UVSX sample satisfy this criterion.

Seven objects (indicated by a star in Table 1) from our sample show a soft X-ray "excess" in the Einstein IPC observations (Masnou et al. 1991). We calculated the soft X-ray (SX) luminosity for these quasars by including only the emission in the soft X-ray excess component. This component, which can dominate at $0.4 \mathrm{keV}$, was derived by subtracting the contribution from the hard energy power law, which is known to be a separate component (Turner \& Pounds 1989). We used the data and spectral parameters given in Masnou et al. (1991).

\subsection{Time Variability and Averaging}

While most of the data were taken between 1978 and 1988 , the full time span is over 25 years, from 1964-1989. A potentially severe limitation on our data set is that the observations are typically not simultaneous, although the optical and ground-based IR data were generally obtained within about 1 month. This problem is worst in the ultraviolet, since the amount of variability increases with frequency throughout the

TABLE 1

UVSX SAMPLE QUASARS

\begin{tabular}{|c|c|c|c|c|c|}
\hline Object $^{2}$ & Name & Host Galaxy & Redshift & V Mag & Class $^{b}$ \\
\hline$Q 0003+158$ & PHL 658 & & 0.450 & 15.60 & RL \\
\hline$Q 0003+199$ & PG $0003+199$ & Mkn 335 & 0.025 & 13.75 & Sy1 \\
\hline$Q 0007+106$ & PG0007+106 & III Zw $2 A$ & 0.089 & 15.16 & RL \\
\hline$Q 0026+129$ & PG0026+129 & PGC 1790 & 0.142 & 15.32 & $\mathbf{R Q}$ \\
\hline Q0049+171 & PG0049+171 & Mkn 1148 & 0.064 & 15.88 & Sy1 \\
\hline$Q 0050+124$ & PG $0050+124$ & I Zw 1 & 0.061 & 14.07 & $\mathbf{R Q}$ \\
\hline Q0052+251 & PG0052+251 & PGC 3237 & 0.155 & 15.42 & $\mathbf{R Q}$ \\
\hline Q0054+144* & PHL 909 & & 0.171 & 16.70 & RQ \\
\hline Q0121-590 & 2E 378 & Fairall 9 & 0.045 & 13.23 & $\mathbf{R Q}$ \\
\hline Q0134+329 & $3 C 48$ & & 0.367 & 16.00 & RL \\
\hline $\mathrm{Q} 0205+024^{*}$ & NAB0205+024 & Mkn 586 & 0.155 & 15.40 & $\mathbf{R Q}$ \\
\hline Q0312-770 & PKS0312-770 & & 0.223 & 15.90 & RL \\
\hline Q0414-060 & $3 C 110$ & & 0.781 & 16.20 & $\mathbf{R L}$ \\
\hline Q0637-752 & PKS0637-752 & & 0.651 & 15.75 & RL \\
\hline Q0804+761 & PG $0804+761$ & & 0.100 & 14.71 & $\mathbf{R Q}$ \\
\hline Q0837-120 & 3C 206 & & 0.198 & 15.76 & $\mathrm{RL}$ \\
\hline Q0844+349 & PG0844+349 & PGC 24702 & 0.064 & 14.00 & $\mathbf{R Q}$ \\
\hline Q0915+165 & Mkn 704 AGN & Mkn 704 & 0.029 & 14.20 & Sy1 \\
\hline Q0923+129 & Mkn 705 AGN & Mkn 705 & 0.028 & 14.60 & Sy1 \\
\hline Q1028+313* & B2 $1028+313$ & & 0.177 & 16.71 & RL \\
\hline Q1100+772 & 3C 249.1 & & 0.311 & 15.72 & $\mathrm{RL}$ \\
\hline Q1116+215 & PG1116+215 & & 0.177 & 15.17 & $\mathrm{RQ}$ \\
\hline Q1137+660 & $3 C 263$ & & 0.652 & 16.32 & $\mathrm{RL}$ \\
\hline Q1146-037 & PKS1146-037 & & 0.341 & 16.90 & RL \\
\hline Q1202+281 & GQ Comae & PGC 38224 & 0.165 & 15.51 & $\mathbf{R Q}$ \\
\hline Q1211+143 & PG1211+143 & PGC 39086 & 0.085 & 14.63 & $\mathrm{RQ}$ \\
\hline Q1219+755* & Mkn 205 AGN & Mkn 205 & 0.070 & 15.24 & $\mathrm{RQ}$ \\
\hline Q1226+023* & $3 C 273$ & PGC 41121 & 0.158 & 12.86 & RL \\
\hline Q1244+026 & PG $1244+026$ & & 0.048 & 16.15 & Sy1 \\
\hline Q1307+085 & PG1307+085 & PGC 45656 & 0.155 & 15.28 & $\mathbf{R Q}$ \\
\hline Q1351+695 & Mkn 279 AGN & Mkn 279 & 0.031 & 14.46 & Sy1 \\
\hline Q1352+183 & PG $1352+183$ & & 0.152 & 15.50 & $\mathbf{R Q}$ \\
\hline Q1407+265 & PG1407+265 & & 0.94 & 15.73 & $\mathbf{R Q}$ \\
\hline Q1416-129 & PG1416-129 & PGC 51142 & 0.129 & 15.40 & $\mathbf{R Q}$ \\
\hline Q1426+015 & PG1426+015 & Mkn 1383 & 0.086 & 15.05 & $\mathbf{R Q}$ \\
\hline Q1501+106* & Mkn 841 AGN & Mkn 841 & 0.036 & 15.09 & Sy1 \\
\hline Q1545+210 & $3 C 323.1$ & & 0.266 & 16.05 & $\mathbf{R L}$ \\
\hline Q1613+658 & PG1613+658 & Mkn 876 & 0.129 & 15.37 & $\mathbf{R Q}$ \\
\hline Q1635+119 & MC2 $1635+119$ & & 0.146 & 16.50 & RL \\
\hline Q1704+608 & $3 C 351$ & & 0.371 & 15.28 & RL \\
\hline$Q 1721+343$ & 4C 34.47 & & 0.206 & 16.50 & RL \\
\hline Q1803+676 & 2E 4008 & Kazaryan 102 & 0.136 & 16.00 & $\mathbf{R Q}$ \\
\hline Q2128-123 & PKS 2128-123 & & 0.501 & 15.46 & $\mathrm{RL}$ \\
\hline Q2130+099* & PG2130+099 & II Zw 136 & 0.061 & 14.62 & $\mathbf{R Q}$ \\
\hline Q2135-147 & PHL 1657 & & 0.200 & 15.53 & RL \\
\hline Q2209+184 & PG 2209+184 & & 0.070 & 15.86 & Sy1 \\
\hline Q2251-178 & MR 2251-178 & & 0.068 & 14.36 & $\mathbf{R Q}$ \\
\hline
\end{tabular}

Asterisks indicate objects with soft X-ray excesses in Einstein IPC(Masnou et al. 1991).

b The classes of object based on optical luminosity and radio spectrum and morphology are RQ (radio-quiet), Sy1 (low-luminosity radio-quiet, or Seyfert 1), RL (radio-loud). 
UVOIR region (Cutri et al. 1985). The magnitude of typical variations is sufficient to contribute to the scatter in the ultraviolet energy distributions.

For about one-third of the objects we have observations at two epochs (occasionally more) in a given wave band, so we can make a crude estimate of the degree of variability. The optical and infrared variability is not a serious problem for these "normal" quasars, but in the ultraviolet the variability is significant on timescales of a few years, although typically it is less than a factor of 2 (Elvis et al. 1994; Kinney et al. 1991).

To generate a single mean energy distribution for each quasar, we have taken an average [in $\log v F(v)]$ of all the data in each frequency bin. This approach gives error bars which account for variability in our nonsimultaneous data. In the UV1/UV2 bands, when the simultaneous data are available the error bars should be much smaller than those we use.

For the IUE data, because of the increased problem of variability and also the widely different $\mathbf{S} / \mathrm{N}$ among observations, we have been selective in the data we chose to include in the average. Specifically, where simultaneous data from the long (LWP/LWR) and short (SWP) wavelength cameras were available, we have included them and excluded "orphan" LWP/LWR or SWP exposures.

\subsection{Luminosities in Individual Bands}

To characterize the large-scale distribution of the energy output of the quasars, we calculate integral luminosities in the set of broad bands defined above (see $\S 2$ ). The IR, VIS, and UV luminosities are the same as in Paper I. The UV1 and UV2 luminosities are new to this paper. The integrals are calculated by running a simple linear interpolation through the data points in $\log v L_{v}$ space, i.e., connecting the individual points with a power law. The errors indicated in the tables are estimated by performing the integrals using the $1 \sigma$ high and $1 \sigma$ low flux values instead of the nominal values. For upper limits, we interpolate between detections on either side. The lower of the interpolated value and the upper limit is used as the nominal flux estimate, but the errors are estimated using zero as the lower error bar and the upper limit as the upper error bar. The logarithms of the calculated integral luminosities in units of $\mathrm{ergs} \mathrm{s}^{-1}$ are tabulated in Table 2.

The colors are determined by taking the logarithm of the ratio between the luminosities within two bands. The estimated uncertainties are a quadrature sum of the errors in the luminosities.

\section{MODELS}

We consider the main models that have been widely discussed in the literature:

1. An accretion disk. We consider the standard $\alpha$-disk models in both Schwarzschild and Kerr geometries (as in Laor \& Netzer 1989; Sun \& Malkan 1989). We include the modification caused by electron scattering and Comptonization of soft photons in the disk atmosphere again for both Schwarzschild (Czerny \& Elvis 1987; Maraschi \& Molendi 1990) and Kerr geometries. We also investigate irradiation of the disk by an external X-ray source (as in Matt, Fabian, \& Ross 1993).

2. Thermal bremsstrahlung (free-free) from a singletemperature optically thin cloud (as in Barvainis 1993).

3 . The combination of the accretion disk and another component: (a) nonthermal power law (as in Czerny \& Elvis 1987; Carleton et al. 1987); (b) thermal emission from a hot dust (as in Sanders et al. 1989; Loska, Szczerba, \& Czerny 1993).
4. The combination of one-temperature thermal bremsstrahlung and another component: (a) nonthermal power law; (b) thermal emission from a hot dust (as in Barvainis 1993).

In the following section we give the details of the construction of each model.

\subsection{Accretion Disk \\ 4.1.1. Local Blackbody Emission}

We assume that the disk radiates locally as a blackbody, so the total flux depends only on the effective temperature distribution Shakura \& Sunyaev 1973). Equations of the disk structure are taken from Novikov \& Thorne (1973) and Page $\&$ Thorne (1974) and include the general relativistic treatment of an accretion disk around a black hole in Schwarzschild and Kerr geometries. General relativistic effects on the propagation of light in the vicinity of the rotating black hole (Cunningham 1975) are calculated using the transfer function tabulated by Laor, Netzer, \& Piran (1990). It takes into account the effect of limb darkening caused by electron scattering in the atmosphere of the disk and the heating of the disk by returning photons (Cunningham 1976).

The inner edge of the disk, $R_{\mathrm{in}}$, is assumed to be the radius of the last marginally stable orbit (e.g., $6 G M / c^{2}$ for a Schwarzschild and $1.23 G M / c^{2}$ for a maximal Kerr black hole, where $M$ is the mass of a black hole). The outer radius $R_{\text {out }}$ of the disk is more difficult to define. The location of $R_{\text {out }}$ determines the frequency at which the emitted flux changes its dependence on frequency, from $v^{2}$, to $v^{1 / 3}$ (Frank, King, \& Raine 1992; Bechtold et al. 1987). Although for large distances the blackbody temperature is low enough to place the characteristic frequency in the IR band, which is usually dominated by other emission components, the chosen value of $R_{\text {out }}$ does affect the computed IR/VIS color of the disk emission (Bechtold et al. 1987). A possible constraint on the extent of the disk might be the radius at which the self-gravity of the disk dominates over the central gravitational force (Laor \& Netzer 1989). However, Störzer (1993) has shown that the effective temperature distribution of a self-gravitating disk is similar to the distribution in the standard disk even if the vertical structures are different. We assumed the same outer radius $2500 R_{\mathrm{S}}\left(R_{\mathrm{S}}=2 G M / c^{2}\right.$ is the Schwarzschild radius) in all our models. For the highest central black hole masses $\left(10^{9} M_{\odot}, 10^{10} M_{\odot}\right)$, this radius is located in the region dominated by the disk gravity.

\subsubsection{Electron Scattering}

For sufficiently high accretion rates, the atmosphere of the disk is dominated by the electron scattering opacity (Shakura $\&$ Sunyaev 1973). Such a spectrum is flatter and much harder than that of local blackbody emission from the disk above the critical frequency $\log v_{\text {crit }} \sim 15.0$ (Novikov \& Thorne 1973; Czerny \& Elvis 1987; Laor \& Netzer 1989; Ross \& Fabian 1993). The modification occurs in the ultraviolet part of the spectrum and affects the UV colors. We calculate the spectrum using the method described by Czerny \& Elvis (1987), which includes modification to the opacity and Comptonization of soft photons caused by the presence of hot electrons in the atmosphere of the disk (Svensson 1984; Czerny \& Elvis 1987). The bound-free opacities are included following the Maraschi \& Molendi (1990) approximation.

\subsection{Irradiation of the Accretion Disk}

$\mathrm{X}$-ray spectra of Seyfert galaxies show signatures of reflection off cold, Compton-thick material (Mushotzky, Done, \& 


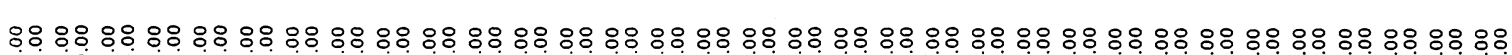

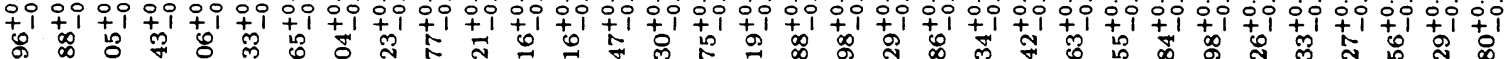
多 稇

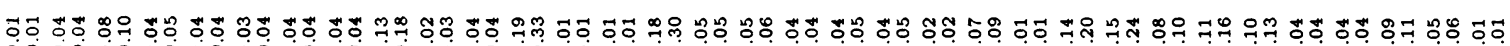

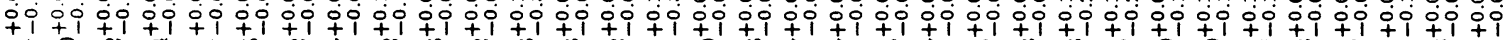

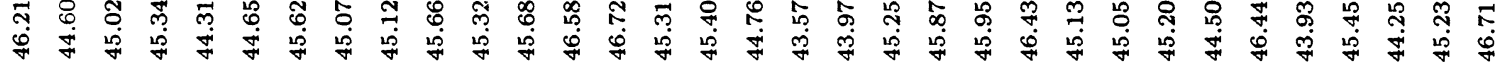

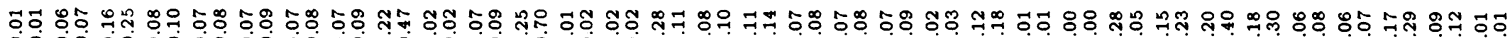

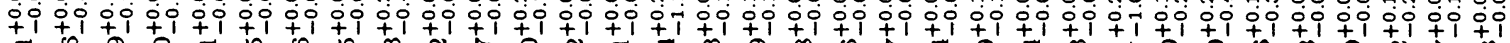
పె 舟

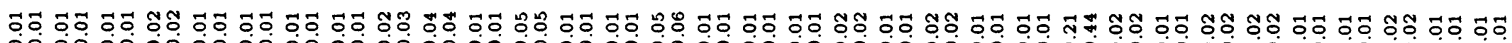

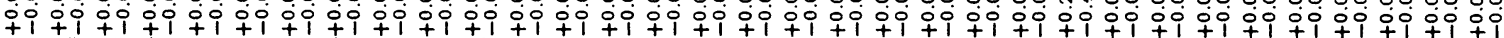

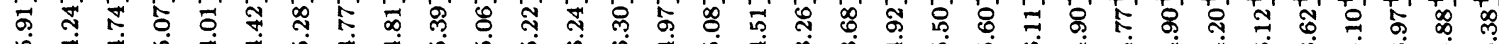
வ

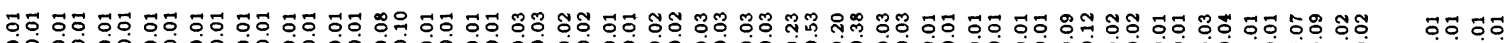

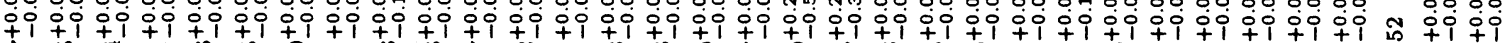

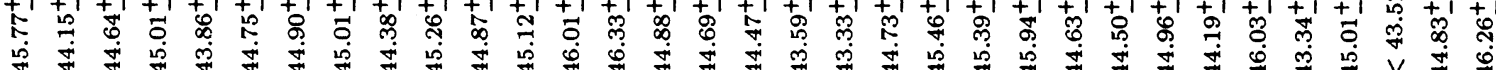

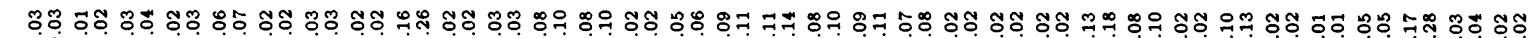

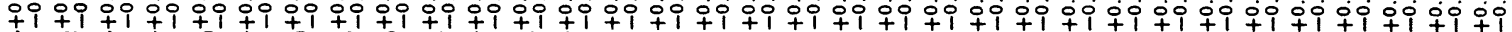

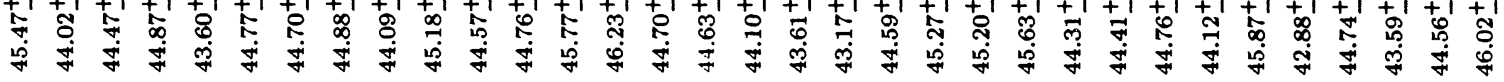




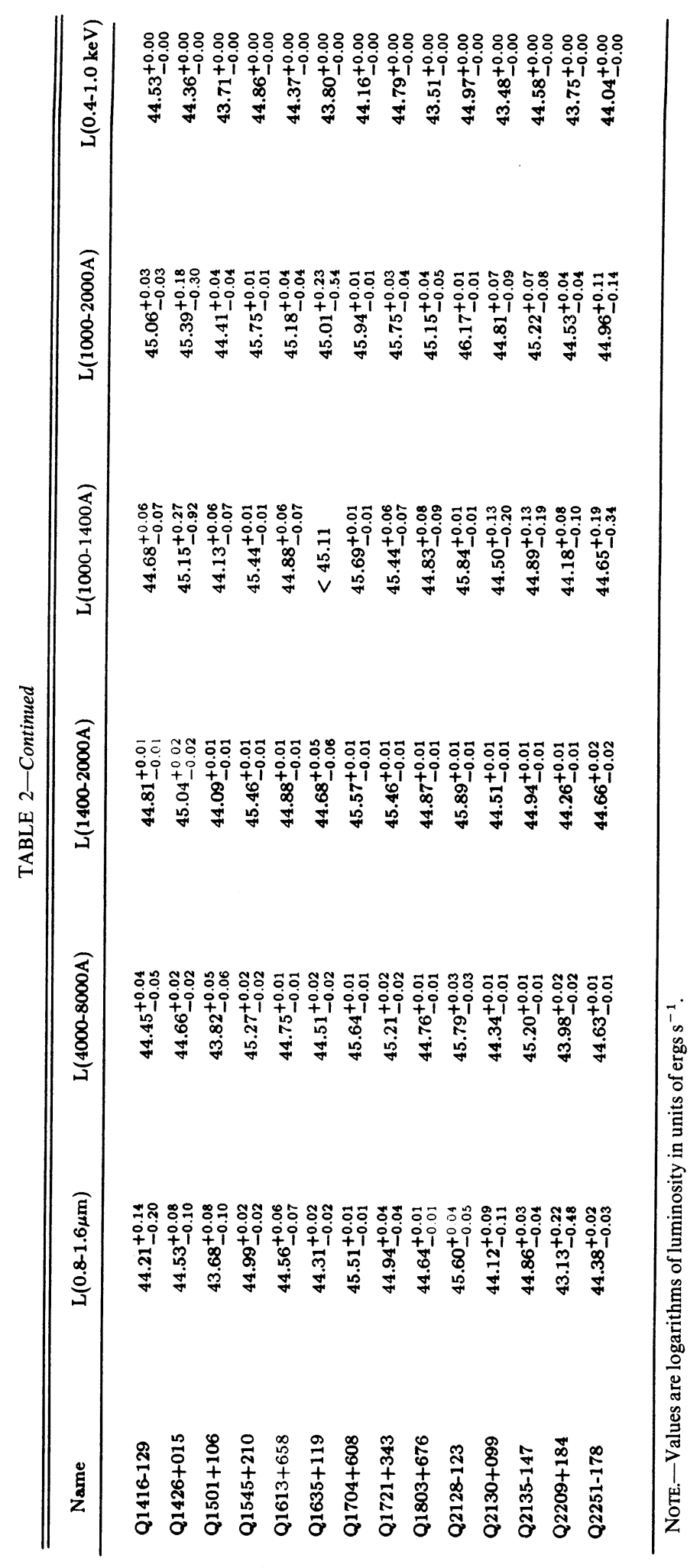


Pounds 1993). While there are other possibilities (e.g., Guilbert \& Rees 1988; Nandra \& George 1994), an accretion disk is a natural source for this mirror.

We assumed that the irradiation gives an extra flux component to the viscously generated local flux. The total local flux is described by (Czerny, Czerny, \& Grindlay 1986; Malkan 1991; Ko \& Kallman 1991; Czerny, Jaroszynski, \& Czerny 1994):

$$
F(R)=F_{0}+\frac{f_{\mathrm{irr}} \eta \dot{M} c^{2}}{4 \pi R^{2}},
$$

where $F_{0}$ is the flux generated in the disk; $\dot{M}$ is the accretion rate in $\mathrm{g} \mathrm{s}^{-1} ; f_{\mathrm{irr}}$ represents the importance of the irradiation in comparison with the total luminosity, describes the efficiency with which the available X-ray flux illuminates the disk including the dependence on the changes in the disk shape, on the angular change to the $\mathrm{X}$-ray source seen by the particular part of the disk, on the scattering in the corona above the disk, etc.; and $\eta$ is the efficiency of converting the gravitational energy into radiation ( $\eta$ is 0.06 for nonrotating and 0.32 for maximally rotating black holes). This approach corresponds to the case in which irradiation is caused by the central X-ray radiation scattered in the corona. The form of equation (1) implies the existence of a critical radius beyond which irradiation dominates the locally generated flux in the disk (Ko \& Kallman 1991). The critical distance can be calculated (we ignore here the relativistic effects, which can cause an increase in the irradiation [Cunningham 1976]):

$$
R_{\mathrm{crit}}=\frac{3 G M}{2 f_{\mathrm{irr}} \eta c^{2}}=\frac{3}{4} \frac{1}{f_{\mathrm{irr}} \eta} R_{\mathrm{S}}
$$

In a simple picture, the whole disk can be divided into two parts: an inner part $\left(R<R_{\text {crit }}\right)$ where the radial effective temperature distribution is dominated by local energy generation [as for the standard disk, $\left.T_{\text {eff }}(R) \sim R^{-3 / 4}\right]$; and an outer part $\left(R>R_{\text {crit }}\right.$ ) where irradiation changes the temperature distribution $\left[T_{\text {eff }}(R) \sim R^{-1 / 2}\right]$. The two parts emit spectra with different slopes: $F_{v}^{\text {in }} \sim v^{1 / 3}$ for the inner part, and $F_{v}^{\text {out }} \sim v^{-1}$ for the outer part. There exists a critical frequency at which the modification to the disk spectrum caused by the irradiation significantly changes the slope of the spectrum compared with that of the original disk. We can calculate this critical frequency from the definition of the blackbody spectrum emitted by each part of the disk using the condition

$$
F_{v_{\text {crit }}}(\text { irr })=F_{v_{\text {crit }}}(\text { disk })
$$

and

$$
F_{v_{\text {crit }}}=\pi \int_{R_{1}}^{R_{2}} B_{v}\left[T_{\text {eff }}(R)\right] R d R,
$$

where $B_{v}$ is the Planck function and the radial temperature distribution is given above. The limits of the integral are given by the inner radius, the critical radius, and outer radius of the disk. Then a comprison between the two parts of the disk with their different temperature distributions gives the critical frequency:

$$
v_{\text {crit }}=1.23 \times 10^{16} M_{8}^{-1 / 4}\left(L / L_{\mathrm{Edd}}\right)^{1 / 4} f_{\mathrm{irr}}^{3 / 4} \eta^{1 / 2} \mathrm{~Hz},
$$

where $M_{8}=M / 10^{8} M_{\odot}, L=\eta \dot{M} c^{2}$ is a luminosity and $L_{\mathrm{Edd}}$ is the Eddington luminosity. Equation (5) shows that irradiation affects the spectrum somewhat more in the case of small central masses. The variability of the high-energy radiation will be correlated with the variability of the optical-UV part of the spectrum for small central mass (and presumably lowluminosity) objects, while for large-mass (high-luminosity) objects, no correlation will be present. For example, in the case of $10^{10} M_{\odot}$ (for irradiation factor $f_{\mathrm{irr}}=0.1, \eta=0.34$, and $\left.L / L_{\text {Edd }}=0.3\right)$ the critical frequency is $\log v_{\text {crit }}=14.47(\sim 1 \mu \mathrm{m})$, and only the spectrum at lower frequencies will be influenced by irradiation, leaving the optical-UV part dominated by the emission from the disk, while for $10^{7} M_{\odot}, \log v_{\text {crit }}=15.22$ ( $\sim 1800 \AA)$, making the optical-UV spectrum influenced by irradiation.

\subsection{Single-Temperature Thermal Bremsstrahlung}

Barvainis (1993) has suggested that the big blue bump is caused by optically thin thermal emission. We consider the simplest such case of single-temperature thermal bremsstrahlung emission from an optically thin $\left\{\tau_{\text {eff }}=\left[\tau_{v}\left(\tau_{v}+\tau_{\text {es }}\right)\right]^{1 / 2}<\right.$ 1 \}, spherical and isothermal cloud. We assume the radius $R$ and density $n_{e}$ of the cloud to be consistent with an optical depth smaller than 1 . The luminosity generated by such a cloud is described by (Rybicki \& Lightman 1979)

$$
L_{v}=9.5 \times 10^{-38} V n_{e}^{2} T^{-1 / 2} \exp \left(-\frac{h v}{k T}\right) g_{\mathrm{ff}} \operatorname{ergs~s}^{-1} \mathrm{~Hz}^{-1}
$$

where $g_{\mathrm{ff}}$ is the Gaunt factor which we approximate using the formula from Gronenschild \& Mewe (1978). The shape of the spectrum is constant, and the peak of the spectrum is shifted to higher frequencies with increasing temperature. Such a cloud emits in the optical UV ( $\left.T \gtrsim 10^{4} \mathrm{~K}\right)$, and for sufficiently high temperatures $\left(T \gtrsim 10^{6} \mathrm{~K}\right)$, it also contributes to the soft X-rays.

\subsection{Additional Components}

Modeling of the observed IR/optical/UV quasar usually requires more than the one emission component. The emission from an accretion disk is not enough to account for the observed power in our IR band. Two main additional components have been considered in the literature: a nonthermal power law, and thermal emission from hot dust heated by the primary continuum. We will consider the direction in which the colors of the basic models can be modified by each of these two components separately. In practice, mixtures of both components may be present.

\subsubsection{Power Law}

A nonthermal power law extending from the far-IR to the $\mathrm{X}$-ray band was considered by a number of authors (Elvis \& Lawrence 1985; Elvis et al. 1986; Carleton et al. 1987; Brissenden 1989; Grossan 1993). There is no compelling evidence for the presence of such a component; however, by assuming such an additional power law it is possible to fit the data over a wide range of frequencies with a minimum of free parameters (Fiore et al. 1995).

We define the power-law normalization by the ratio $L_{2000 \AA} / L_{3 \mu \mathrm{m}}$. We use the spectral index $\left(v L_{v} \sim v^{-\alpha_{\text {OIR }}}\right) \alpha_{\text {OIR }}=$ $1.23[ \pm 0.28(1 \sigma)]$ given by the averaged ratio for our sample (Paper I). We discuss the consequences of changing the powerlaw spectral index and normalization on the calculated colors. 


\subsubsection{Hot Dust \\ 4.4.2.1. Optically Thick}

There are observational indications that hot dust is located in the nuclear region (e.g., Fairall 9 in Clavel et al. 1992; NGC 3783 in Glass 1992; GQ Comae in Sitko et al. 1993). Assuming that the luminosity of the big blue bump provides the flux irradiating the dust, we analyze the variations in colors caused by emission from a hot, optically thick torus of dust. Since the central continuum is the main source of energy for the dust, its effective temperature at a certain radius depends only on its distance from the continuum source (Barvainis 1990; Laor \& Draine 1993). We adopt the relation given by Laor \& Draine (1993) for an optically thick, plane-parallel dust slab:

$$
T_{\text {eff }}(R)=T_{\max }\left(\frac{R}{R_{\mathrm{in}}}\right)^{-1 / 2}
$$

where $T_{\max }$ is the maximum dust temperature at the inner radius, $R_{\text {in }}$, of the dust. The minimum radius of the hot dust is defined (for a given central luminosity) by the sublimation temperature of the grains present and depends on their size and the chemical composition. This temperature ranges from $\sim 900 \mathrm{~K}$ for silicon to $\sim 1750 \mathrm{~K}$ for mixtures of graphite grains. We calculated the spectra emitted by the hot dust assuming evaporation temperatures ranging from $900 \mathrm{~K}$ to $1750 \mathrm{~K}$ to define the inner radius of the dust. We assume that the dust is optically thick, and we calculate the blackbody emission from a flat disk viewed face-on. This gives a maximum dust contribution at the near-infrared frequencies. The outer radius of the dust is defined by the lower temperature cut which we take to be 250 $K$, to put the cutoff well outside the IR band. We examine the two-component models in the color-color diagrams.

\subsubsection{Optically Thin}

The hot dust in the central parsec can also form an optically thin shell around the central continuum source, causing absorption of the optical-UV continuum (Laor \& Draine 1993; Loska et al. 1993). The spectrum emitted by the disk (or other source) is reprocessed by the shell of dust. The emergent spectrum depends on the continuum input spectrum, the chemical composition of the dust, the size of dust grains, the size of the dust region, and the disk inclination angle. In general, our UV band will be affected slightly more than the VIS band. We do not calculate this model, since it requires a detailed treatment of the radiation transfer through the dust, which we do not consider. In Figures 2 and 3 we show the vector pointing in the direction the colors would change as a result of the presence of the optically thin dust. The vector shows the reddening given by Laor \& Draine (1993) for a spherical shell of dust $($ graphite $+\mathrm{SiC})$ with $E(B-V)=0.033$.

\section{COLOR-COLOR, COLOR-LUMINOSITY DIAGRAMS}

We discuss the color-color and color-luminosity diagrams for each group of models. The primary diagram is that of VIS/UV1/UV2 because it is most sensitive to the slope of the big blue bump.

\subsection{Thermal Bremsstrahlung}

The spectrum of a hot, optically thin plasma emitting freefree depends on its temperature (eq. [6]) and has a well-defined shape. We show the change in the VIS/UV1/UV2 colors

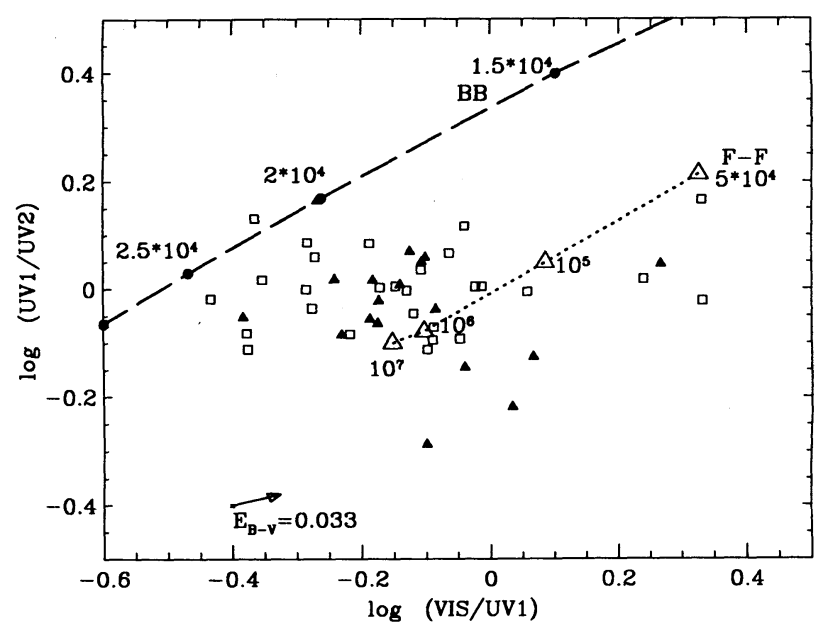

FIG. 2.-VIS/UV1/UV2 color diagram. Lines represent different models. Dotted lines single-temperature free-free emission, empty triangles on the dotted line indicate the temperatures $\left(5 \times 10^{4}, 10^{5}, 10^{6}\right.$, and $\left.10^{7} \mathrm{~K}\right)$; dashed line: single-temperature blackbody (filled circles show the temperatures) Radio-loud quasars are indicated by filled triangles and radio-quiet quasars are indicated by open squares. The vector of modifications expected as a result of the hot optically thin dust for $E(B-V)=0.033$ is indicated by an arrow.

resulting from variations in temperature as the dotted line in Figure 2, which also shows the data points. (Error bars that also allow for variability [ $\$ 3.1]$ are plotted in Fig. 7.) We indicate radio-loud objects with solid triangles and radio-quiet with empty squares. There is no difference in the scatter of the radio-loud and radio-quiet objects in this diagram. The data points are distributed over a larger region than the simple free-free emission can produce.

While optically thin plasmas with temperatures between $5 \times 10^{4}$ and $5 \times 10^{7} \mathrm{~K}$ produce spectra within the observed range of UV1/UV2 colors, they do not simultaneously give the VIS/UV1 colors required by the observations. The theoretical spectra predict a continuum shape which is too flat for some objects and the VIS/UV1 is too large (Fig. 2). Adding a nonthermal power-law component mainly increases the VIS luminosity and gives a possible explanation for some of the data points. But the objects with VIS/UV1 color smaller than that of our free-free emission [below $\log (\mathrm{VIS} / \mathrm{UV} 1) \sim-0.15$ ] are not reachable by this means. The dashed line in Figure 2 shows the colors of the single-temperature blackbody emission. Moderate optical depths $(0<\tau<$ few $)$ can populate the region between the blackbody and free-free lines (Collin-Souffrin et al. 1995). Objects with large VIS/UV1 color cannot be accommodated, however, and several of these objects have colors that place them securely in this region.

We conclude that an optically thin bremsstrahlung-emitting plasma at a single temperature is not an appropriate model for the optical/UV part of the big blue bump, at least for our sample.

\subsection{Accretion Disks}

We calculated colors for the following model parameters: black hole masses $\left(10^{6} M_{\odot}, 10^{7} M_{\odot}, 10^{8} M_{\odot}, 10^{9} M_{\odot}\right.$, and $\left.10^{10} M_{\odot}\right)$, accretion rates $\left(0.01 \dot{M}_{\text {Edd }}, 0.1 \dot{M}_{\text {Edd }}, 0.3 \dot{M}_{\text {Edd }}\right.$ and $0.8 \dot{M}_{\text {Edd }}$, where $\dot{M}_{\text {Edd }}$ is an accretion rate which corresponds to the Eddington luminosity), and inclination angles ( $\cos \theta=$ $0.1,0.25,0.5,0.75$, and 1 ). 


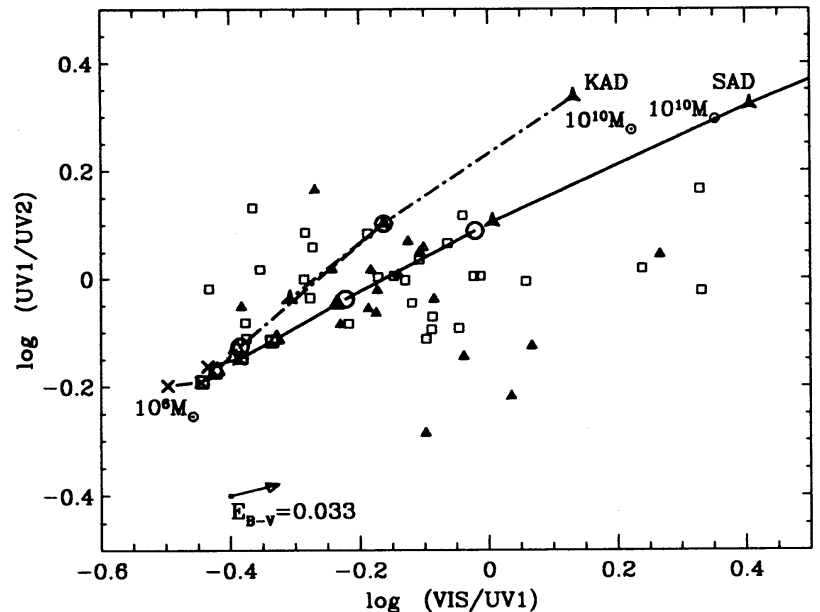

Fig. 3.-VIS/UV1/UV2 color diagram. Lines represent different models. Solid line: accretion disk in Schwarzschild (SAD) geometry; dash-dotted line: accretion disk in Kerr (KAD) geometry, both with local blackbody emission for a range of central black hole masses $\left(10^{6}-10^{10} M_{\odot}\right)$ and accretion rates $\left(0.01-0.8 \dot{M}_{\mathrm{Edd}}\right)$, for one inclination angle $\cos \theta=0.75$. The two values of accretion rates, $0.1 \dot{M}_{\odot}$ and $0.8 \dot{M}_{\odot}$ are shown for each mass (crosses for $10^{6} \mathrm{M}_{\odot}$, squares for $10^{7} M_{\odot}$, triangles for $10^{8} M_{\odot}$, circles for $10^{9} M_{\odot}$, and three angle stars for $10^{10} M_{\odot}$ : for this mass, we also give the $0.01 \dot{M}_{\text {Edd }}$ point). Radio-loud quasars are indicated by filled triangles, and radio-quiet quasars are indicated by empty squares. The vector of modifications expected as a result of the hot optically thin dust for $E(B-V)=0.033$ is indicated by an arrow.

\subsubsection{Degeneracy between Mass and Accretion Rate in the Standard Disk Models}

Figure 3 shows, on the same scale as Figure 2, the loci of the standard accretion disk models emitting locally as a blackbody represented by the solid (Schwarzschild) and dash-dotted (Kerr) lines in VIS/UV1/UV2 colors. The marks on the lines mark different central masses and accretion rates at a constant inclination angle, $\cos \theta=0.75$. A degeneracy between mass and accretion rate is visible: the same point on the curve can be explained by different combinations of central mass and accretion rate. This degeneracy can be understood quite simply. The shape of the spectrum is defined by the combination of mass and accretion rate $\left(\dot{M} / M^{2}\right)$ (where $\dot{M}$ is in units of $M_{\odot} \mathrm{yr}^{-1}$ ), which is related to the characteristic frequency at which the spectrum peaks in a $v F_{v}$ plot. It can be shown (Novikov \& Thorne 1973; Czerny \& Elvis 1987) that the total radiated spectrum peaks at the frequency

$$
\log v_{\text {peak }}=19.55+0.25 \log \left(\frac{\dot{M}}{M^{2}}\right)
$$

for the Schwarzschild geometry and at

$$
\log v_{\text {peak }}=20.07+0.25 \log \left(\frac{\dot{M}}{M^{2}}\right)
$$

for the Kerr geometry $\left(M\right.$ is in $M_{\odot}$ and $\dot{M}$ is in $\left.M_{\odot} \mathrm{yr}^{-1}\right)$. This is only true for disks emitting locally as blackbodies. The shape is then constant, determined by the combination of mass and accretion rate, and only the peak moves with the frequency.

Figure 3 shows also the data. As for the free-free model, the simplest disk model is represented by a line in the color-color diagram and cannot reproduce the full range of colors, especially these at large VIS/UV1. However, we need to include more physics in the disk models (Czerny 1994). We consider some additional physics below.

\subsubsection{Inclination}

Disk inclination changes the observed colors strongly because of the effects of general relativity. The changes caused by inclination in the Kerr geometry are much larger than in the Schwarzschild case because the disk extends to smaller numbers of Schwarzschild radii. We show only the Kerr case below. Figure 4 shows the spread in VIS/UV1/UV2 colors resulting from changing the disk inclination angle in the Kerr geometry $\cos \theta=(0.1,0.25,0.5,0.75$, and 1$)$, where 0.1 corresponds to the disk seen almost edge-on, and 1 corresponds to face-on.

The range of colors covered is much wider at large $M$ $\left(\sim 10^{10} M_{\odot}\right)$ than at small $M\left(\sim 10^{7} M_{\odot}\right)$. However, there is no combination of mass and accretion rate which gives a spread in colors away from the narrow region in Figure 4. Changes in inclination do not help to produce the observed colors.

\subsubsection{Electron Scattering and Comptonization}

In Figures 5 and 6 we plot model loci in the VIS/UV1/UV2 colors when the electron scattering and Comptonization in the disk atmosphere for two geometries are included. The degeneracy between accretion rate and central mass now disappears. For higher accretion rates $(\dot{M} \gtrsim 0.1)$, the models move away from the line defined for local blackbody emission from the disk (Fig. 3). The opacity caused by free electrons is more important for high accretion rates when the disk is hot, while for $\dot{M} \lesssim 0.1 \dot{M}_{\text {Edd }}$ there is no change in the colors compared to a disk radiating a local blackbody.

The modification resulting from electron scattering and Comptonization moves low-energy photons (mainly blue and UV) to higher frequencies (extreme UV and soft X-ray). This flattens the spectrum (in $v L_{v}$ sense) and makes the turnover in the far-UV less sharp. The resulting spectrum covers a much wider range of colors. For large masses $\left(\gtrsim 10^{9} M_{\odot}\right)$, the change (caused by free electrons) of the VIS/UV1 color in comparison with the local blackbody disk is larger than for the small central mass $\left(\$ 10^{8} M_{\odot}\right)$, where mainly the UV1/UV2 color is modified. This is because a disk around a small central mass

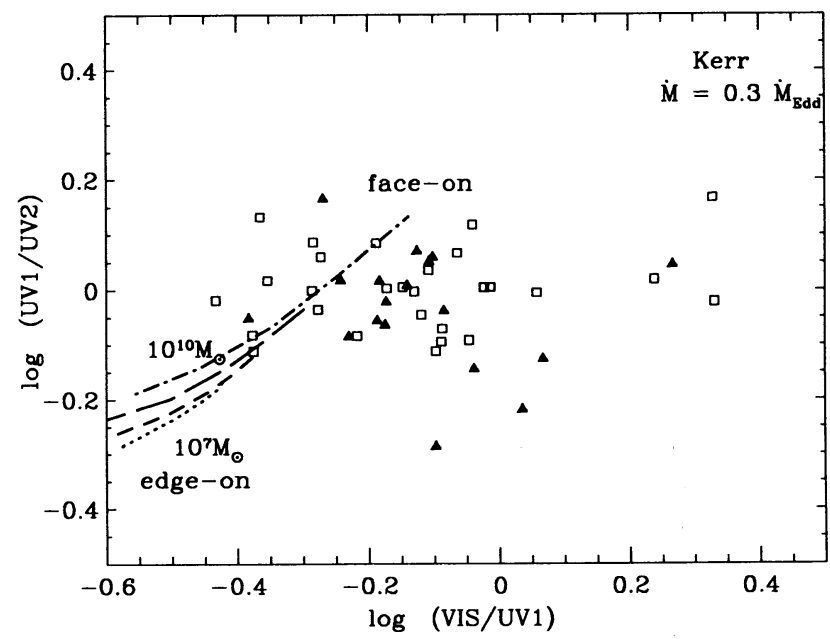

Fig. 4.-The colors of the accretion disk (Kerr) emission (constant accretion rate $\dot{M}=0.3 \dot{M}_{\mathrm{Edd}}$ ) when the inclination angle is changed from edge-on to face on at different central masses $10^{7} M_{\odot}$ (dotted line), $10^{8} M_{\odot}$ (short-dashed line), $10^{9} M_{\odot}$ (long-dashed line), and $10^{10} M_{\odot}$ (dot-dashed line). Radio-loud quasars are indicated by filled triangles, and radio-quiet quasars are indicated by open squares. 


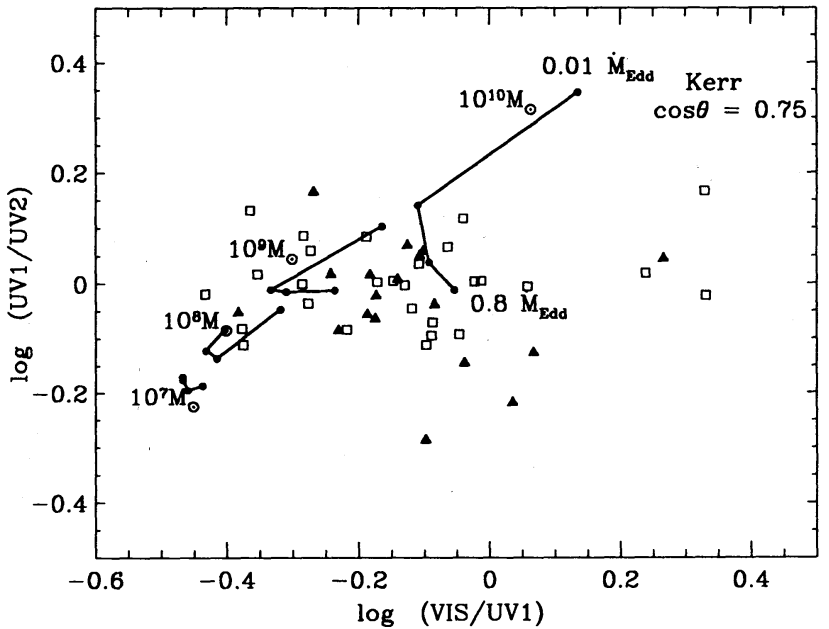

FIG. 5.-The colors of the modified blackbody accretion disk (Kerr) for different mass $\left(10^{7}-10^{10} M_{\odot}\right)$ and accretion rates $\left(0.01-0.8 \dot{M}_{\mathrm{Edd}}\right)$, but constant inclination angle $(\cos \theta=0.75)$. Radio-loud quasars are indicated by filled triangles and radio-quiet quasars are indicated by open squares.

has a higher maximum temperature for the same accretion rate than that around a large black hole. It peaks in the far-UV, and Comptonization modifies that part of the spectrum which lies outside the discussed bands.

Figures 5 and 6 show also the data. The modified blackbody range of VIS/UV1/UV2 colors spans the data better, particularly for lower black hole masses in a Schwarzschild geometry. Depending on the geometry, the best range of the central mass is between $10^{7} M_{\odot}-10^{8} M_{\odot}$ for a Schwarzschild geometry and $10^{8} M_{\odot}-10^{10} M_{\odot}$ for a Kerr geometry. There are still objects with very high VIS/UV1 color which cannot be explained by this model.

\subsubsection{Summary of Disk Models: Outlying Points}

So far we have discussed only the VIS/UV1/UV2 colors, which are the most important for studying the accretion disk

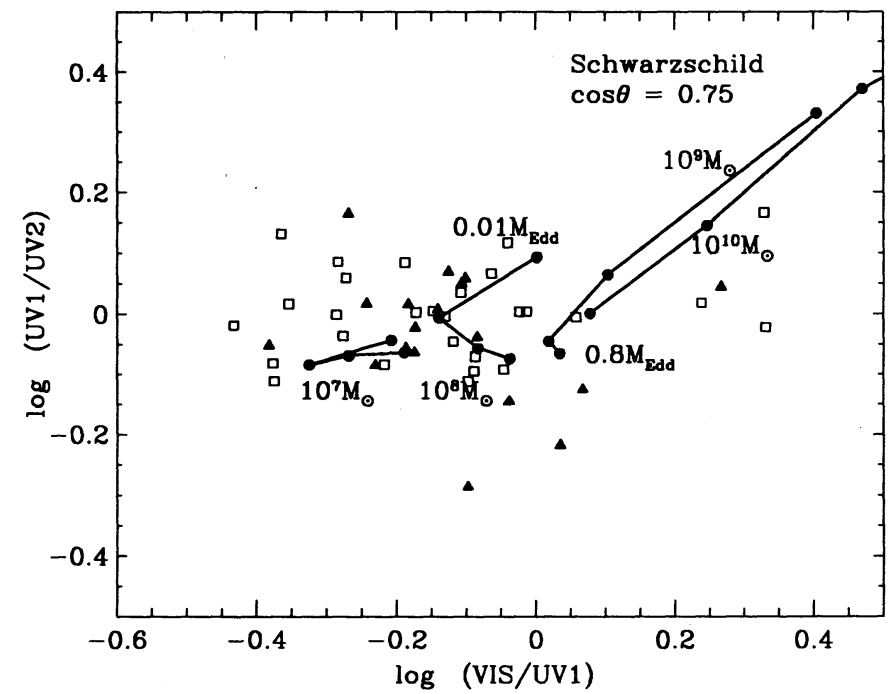

FIG. 6.-The colors of the modified blackbody accretion disk (Schwarzschild) for different mass $\left(10^{7}-10^{10} M_{\odot}\right)$ and accretion rates $\left(0.01-0.8 \dot{M}_{\text {Edd }}\right)$, but constant inclination angle $(\cos \theta=0.75)$. Radio-loud quasars are indicated by filled triangles, and radio-quiet quasars are indicated by open squares. models. We show the area of the VIS/UV1/UV2 plane covered by accretion disk models in Figure 7. We also plot the data for our sample of quasars including conservative error bars which account also for variability (see $\S 3.1$ ). There are two regions of colors corresponding to accretion disk models in Schwarzschild and Kerr geometries. The areas include the colors reachable for any combination of the accretion disk parameters (central mass, accretion rate, inclination). Most of the data points are located inside the regions covered by the models, which is generally encouraging for disk models. However, there are 10 objects outside both regions, and modifications to a pure accretion disk are needed to explain these points. It is important to be sure there is no problem with the data in these cases. In particular, the errors on the UV1/UV2 color resulting from the $I U E$ observations are usually large, and there is the problem of the nonsimultaneity of the optical and UV observations which affects the VIS/UV1 color.

Also interesting is a group of four of the 10 objects with a high VIS/UV1 color. These are so called "weak bump" quasars (McDowell et al. 1989). In each of the following figures, they form a separate group. PHL 909 is a highluminosity, radio-quiet quasar. PHL 1657 (PKS 2135-147) is a radio-loud object in a rich cluster. Mkn 705 and $I \mathrm{Zw} 1$ are lower luminosity active nuclei; Mkn 705 in particular is affected by uncertainties in host galaxy starlight subtraction. Correct starlight subtraction has moved Mkn 876, previously suggested to be a weak bump quasar (McDowell et al. 1989), into the central group of objects.

Positive values of $\log (\mathrm{UV} 1 / \mathrm{UV} 2)$ indicate the presence of the turnover in the spectrum. The frequency of the turnover is important since it gives information on the mass and accretion rate for a particular accretion disk model. From equations (8)-(9) and the definition of UV1 and UV2 bands, the range of appropriate combinations for the turnover to be located inside the color band is between $-17.48 \lesssim \log \left(\dot{M} / M^{2}\right) \lesssim-16.3$ for the Schwarzschild and $-19.56 \lesssim \log \left(\dot{M} / M^{2}\right) \lesssim-18.36$ for the Kerr disks.

These are a few objects with positive UV1/UV2 colors (Mkn 589, PHL 909, Q0050+124, Q0134+329, Q1803+676, and $\mathrm{Q} 0026+129)$. However, all of them have large uncertainties,

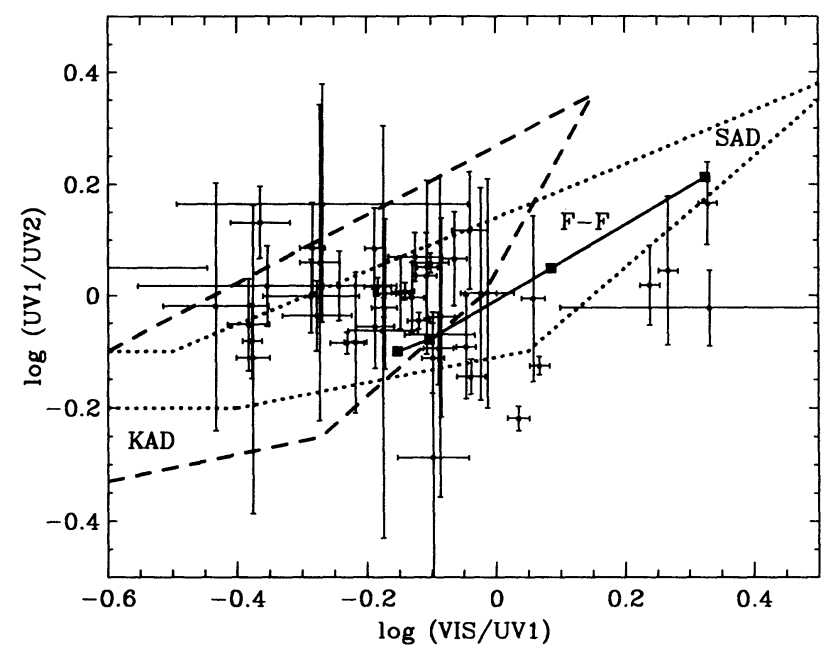

FIG. 7.-The regions of colors covered by pure Schwarzschild (SAD, inside dotted line region) and $\mathrm{Kerr}(\mathrm{KAD}$, inside long-dashed region) accretion disk models. The single-temperature free-free model for temperatures as in Fig. 2 is indicated by the solid line (F-F). 
and the turnover is not clearly visible in their spectra. All objects with positive values are interesting for further studies, since they are the most probable candidates for observations of a turnover. The turnover may also be caused by the internal absorption as may happen in the BAL quasar PG 1416-129. This quasar has small error bars and a high UV1 luminosity and it is the best candidate to study.

Radio-loud and radio-quiet objects cover the same range of colors.

\subsubsection{Color-Luminosity Diagram}

Luminosity gives an extra constraint which allows us to recognize better the features of the models and understand their capability to reproduce observations. For example, the mass accretion rate degeneracy present in the color-color plots (Fig. 3) for local blackbody emission from accretion disks disappears partially in the color-luminosity diagram. Nevertheless, there are still regions of color-luminosity space in which a single point may be modeled by several different combinations of central mass, accretion rate, and inclination.

Figures 8 and 9 show the UV1/UV2 color versus luminosity in the UV2 band $\left(L_{\mathrm{UV}_{2}}\right)$. This choice of color is similar to that used by Caditz (1993). In Figure 8 we plot the models for the accretion disk with local blackbody emission in the Kerr geometry. The change in the inclination for each mass with constant accretion rate $\left(\dot{M}=0.8 \dot{M}_{\text {Edd }}\right)$ is indicated by the open symbols. The change caused by different accretion rates for a given inclination angle $(\cos \theta=0.75)$ is indicated by the filled symbols.

In Figure 9 we show the modified blackbody emission from the accretion disk in the Kerr geometry. The UV1/UV2 colors are higher, which corresponds to flattening of the spectrum in this band. The data points are all located well inside the region described by blackbody disk models. Large masses for the central black hole are preferred, but in general the agreement is surprisingly good. However, the UV1/UV2 color spans only a very narrow frequency region covering only the peak of the big blue bump. Interestingly, the local blackbody emission seems

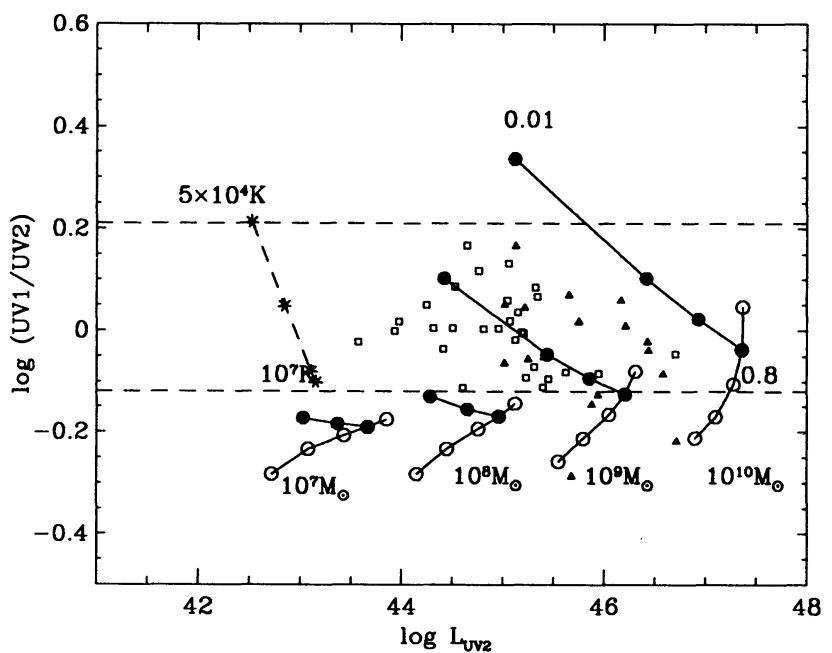

FIG. 8.-UV1/UV2 color vs. luminosity (UV2) for local blackbody disk models (Kerr). Open symbols show the effect of the inclination for the constant accretion rate $\left(\dot{M}=0.8 \dot{M}_{\text {Edd }}\right)$. Filled symbols indicate the colors and luminosities for different accretion rates but one inclination $(\cos \theta=0.75)$. Dashed lines indicate the range of UV1/UV2 color for free-free emission. Stars show the observed colors and luminosities.

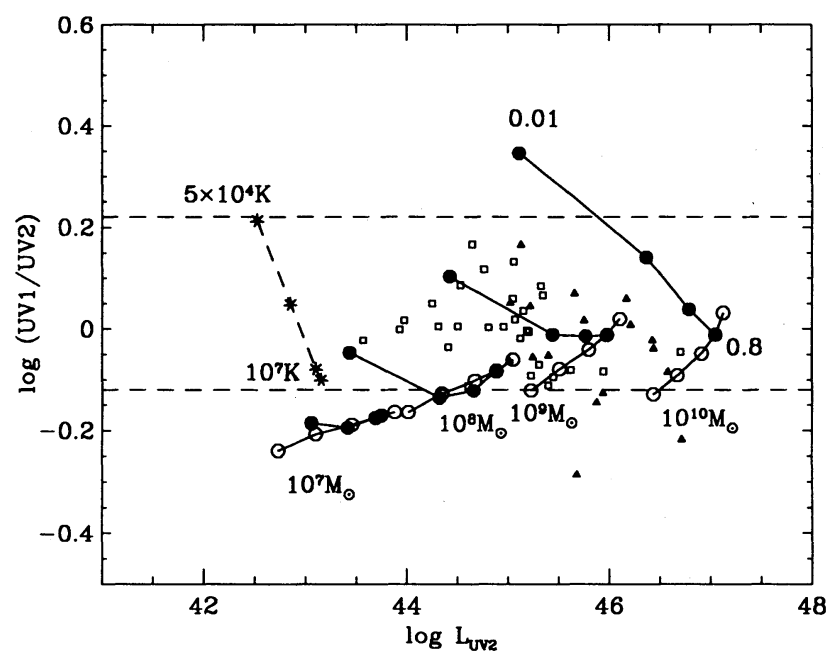

FIG. 9.-UV1/UV2 color vs. luminosity (UV2) for modified blackbody disk models (Kerr). Open symbols show the effect of the inclination for the constant accretion rate $\left(M=0.8 \dot{M}_{\text {Edd }}\right)$. Filled symbols indicate the colors and luminosities for different accretion rates but one inclination $(\cos \theta=0.75)$. Dashed lines indicate the range of UV1/UV2 color for free-free emission. Radio-loud quasars are indicated by filled triangles, and radio-quiet quasars are indicated by open squares.

to explain the data better than the modified blackbody. However, by comparing this figure to the color-color diagram (VIS/UV1/UV2, Fig. 3), it is clear that while UV1/UV2 is well described by the disk model, the VIS/UV1 color is not.

These two figures are the very similar to diagrams in Tripp et al. (1994) (also Wandel \& Petrosian 1988), which were useful for studying variability patterns of two Seyfert galaxies, NGC 5548 and NGC 3782. Tripp et al. show that the observed variability is consistent with changing an accretion rate and constant central black hole masses for both objects. Our data allow us to make a similar analysis for 3C 273. In Figure 10 we plot all available data for this quasar binned in $1 \mathrm{yr}$ periods. The variability path of $3 \mathrm{C} 273$ is consistent with changes in accretion rates and the constant mass. However, it is also consistent with temperature changes in the free-free model.

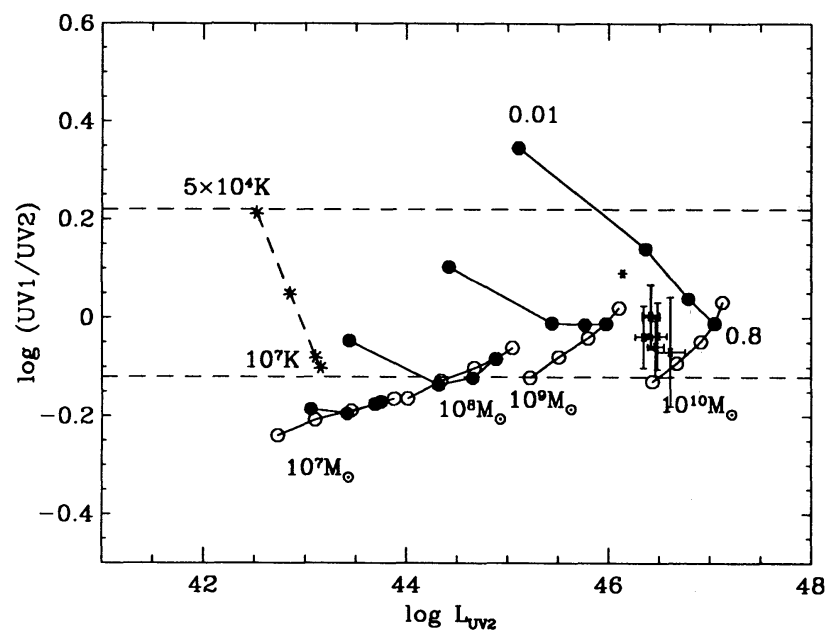

Fig. 10--UV1/UV2 color vs. luminosity (UV2) showing the modified blackbody disk models as in Fig. 9. Stars indicate 3C 273 data points binned into $1 \mathrm{yr}$ periods. 
The VIS/UV color covers a wider range of frequencies. We plot the relation between the VIS/UV color and the UV luminosity for accretion disk models in the Kerr geometry in Figure 11. Different central masses are shown in the figure $\left(10^{7}\right.$ $\left.M_{\odot}-10^{10} M_{\odot}\right)$. Open circles represent the range of change in color and luminosity caused by inclination for a given accretion rate $\left(\dot{M}=0.8 \dot{M}_{\text {Edd }}\right)$. The change caused by accretion rate variations is indicated by the filled circles and is plotted for one inclination only $(\cos \theta=0.75)$. The area covered by accretion disk models for a big black hole mass $\left(\gtrsim 10^{9} M_{\odot}\right)$ is much larger than for small masses. Models with large central mass and low accretion rate can explain most of the data points located in this plot.

Color-color diagrams do not require the information about cosmological parameters for the model colors, since we use rest frame luminosities. However, the calculated object luminosities used in color-luminosity diagrams do require an assumption on $H_{0}$ and $\Omega_{0}$. Since we considered only low-redshift objects, there is no visible difference between various parameter values. However, for high-redshift objects the change in parameter values causes a large change in the luminosities. We indicate in Figure 11 the direction of the expected luminosity change for $z=0.5$ when $H_{0}=100 \mathrm{~km} \mathrm{~s}^{-1} \mathrm{Mpc}^{-1}$ and $\Omega_{0}=0$.

\subsection{Irradiation of the Accretion Disk}

When the accretion disk is irradiated by an external X-ray source, the effective temperature distribution is modified. For typical parameters, this causes significant changes only in the VIS band luminosity. The range of colors available for the irradiated disk is indicated in Figure 12 by two solid lines with the filled squares. The points indicate the central mass and accretion rate which are the same as in the pure disk case. The largest modification is expected for large central mass and quite large fraction of the irradiating flux. However, even small irradiation gives much more flexibility to the model than the emission from the hot dust (see $\S$ 5.4.2).

Figure 13 shows the change in VIS/UV1/UV2 colors resulting from irradiation of the disk by an external source.

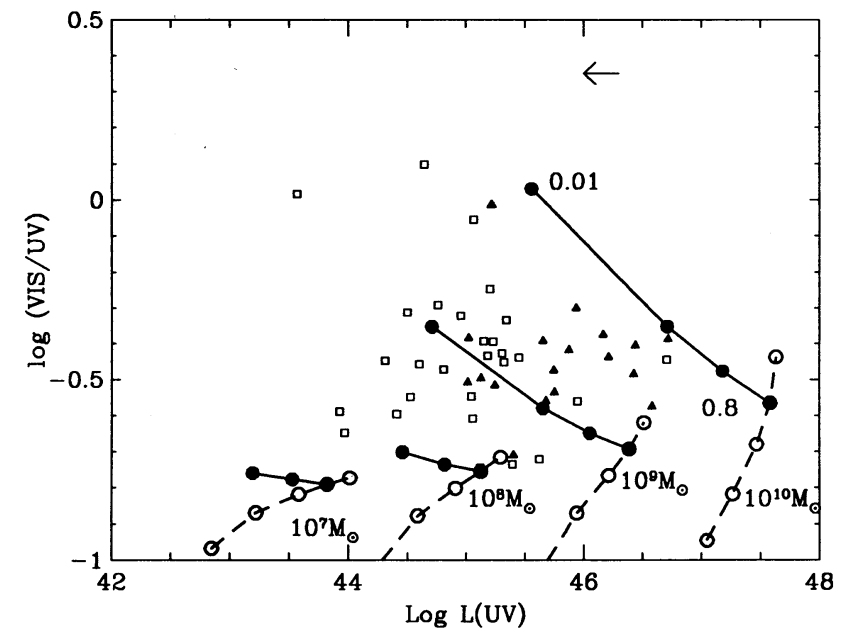

FIG. 11.-VIS/UV vs. UV luminosity local blackbody emission from accretion disk (Kerr). Open symbols show the effect of the inclination for the constant accretion rate $\left(\dot{M}=0.8 \dot{M}_{\mathrm{Edd}}\right)$. Filled symbols indicate the colors and luminosities for different accretion rates but one inclination $(\cos \theta=0.75)$. The arrow shows the change in the luminosity caused by the difference in $H_{0}$ and $\Omega_{0}$ for an object at $z=0.5$. Radio-loud quasars are indicated by filled triangles, and radio-quiet quasars are indicated by open squares.

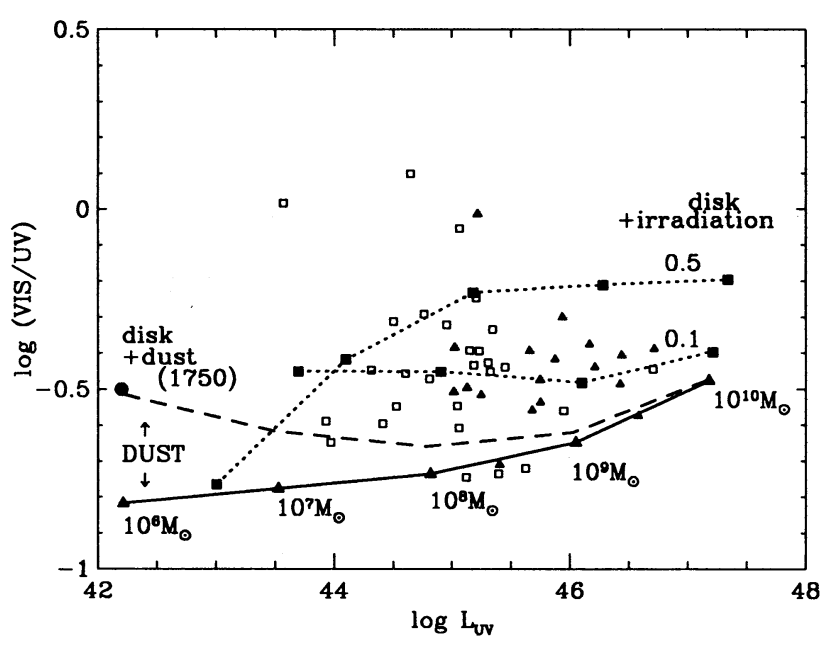

FIG. 12.-VIS/UV vs. UV luminosity diagram. The lower solid lines with filled triangles indicate the emission from the pure accretion disk model (Kerr, local blackbody) for different mass and the same accretion rate $\left(\dot{M}=0.3 \dot{M}_{\text {Edd }}\right)$ and inclination angle ( $\cos \theta=0.75$ ). Dashed line shows modification caused by the hot dust with the maximum temperature of $1750 \mathrm{~K}$. Dotted lines with the filled squares show the effect of irradiation. Radio-loud quasars are indicated by filled triangles, and radio-quiet quasars are indicated by open squares.

The largest modification to the colors compared to pure disk colors is in the VIS/UV1. This is because the outer parts of the disk (with the correct effective temperatures to contribute to the $V$-band luminosity) are irradiated the most. The VIS/UV1 color changes more than the UV1/UV2, showing the importance of the irradiation at lower frequencies. For low central masses even a small fraction of irradiation $(<0.1)$ changes the VIS/UV1 color significantly. For large masses, the effect can be noticed only when the irradiated flux is strong (e.g., $\approx 0.5$ in our parameterization).

The limit for our model is given by the critical radius. For a given value of irradiated flux and parameters of the disk, the critical radius becomes smaller than the inner radius of the disk. Then the entire disk surface is irradiated significantly, and

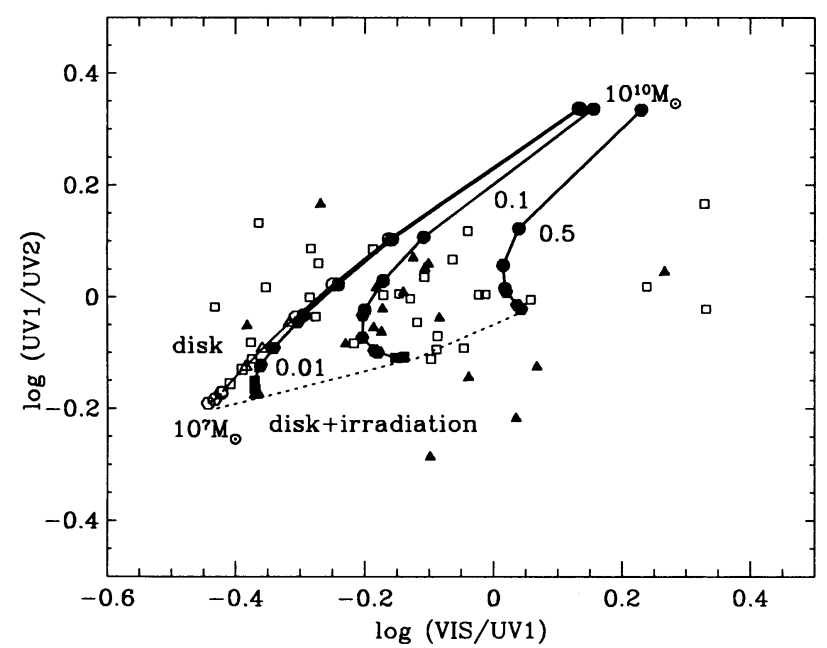

Fig. 13.-VIS/UV1/UV2 colors. Modifications to the pure disk colors (local blackbody) as a result of irradiation are shown for three values of irradiation factor $f_{\text {irr }}: 0.01,0.1$, and 0.5 . Dotted line indicates the limit caused by the critical radius. Radio-loud quasars are indicated by filled triangles, and radio-quiet quasars are indicated by open squares. 
our simple approach is not valid. We indicate the maximum allowed color change by the dotted line in Figure 13.

\subsection{Additional Components}

While the luminosity from an accretion disk can produce all the optical, ultraviolet, and possibly that soft-X-ray part of the spectrum (see $\S 5.5$ ), it is not sufficient to account for the nearinfrared emission. Other sources of emission must be added when extending the modeling of quasar continua into this range. Thermal dust emission from a torus in the nuclear region and nonthermal power laws are the extra components most discussed (Elvis \& Lawrence 1985; Carleton et al. 1987; Fiore et al. 1995; Laor \& Draine 1993).

\subsubsection{Additional Power Law}

Figure 14 shows how the VIS/UV1 and UV1/UV2 colors are modified when an underlying power law is added to the accretion disk spectrum (Kerr, local blackbody emission). The directection of changes in the colors resulting from different power-law normalizations and spectral indices is indicated. The main change is in the VIS/UV1 color and is caused by an increase in VIS luminosity. Adding the power law to the freefree emission moves the free-free line in the same direction as in the disk case.

The changes in the longer wavelength VIS/UV/IR colors are larger. Figure 15 shows the relation between VIS/UV and UV/IR model colors. All four types of models (single blackbody, free-free, sum of blackbodies, and modified blackbodies) generate a similar continuum in this wide range of frequencies and are all represented by the solid line. Naturally, the location along the line depends on the parameters of the particular model. A large mass gives lower UV/IR colors than a small central mass. The points on the line can be represented by a combination of central mass and accretion rates. We have calculated the range of accretion rates corresponding to $0.01-0.08 \dot{M}_{\text {Edd }}$.

In Figure 15 we indicate the change in colors resulting from adding a nonthermal power-law to the accretion disk emission or free-free emission. Spectral slopes and normalizations of the power-law are indicated in Figure 15. The change is strong and clear for the IR band. The magnitude of this change depends

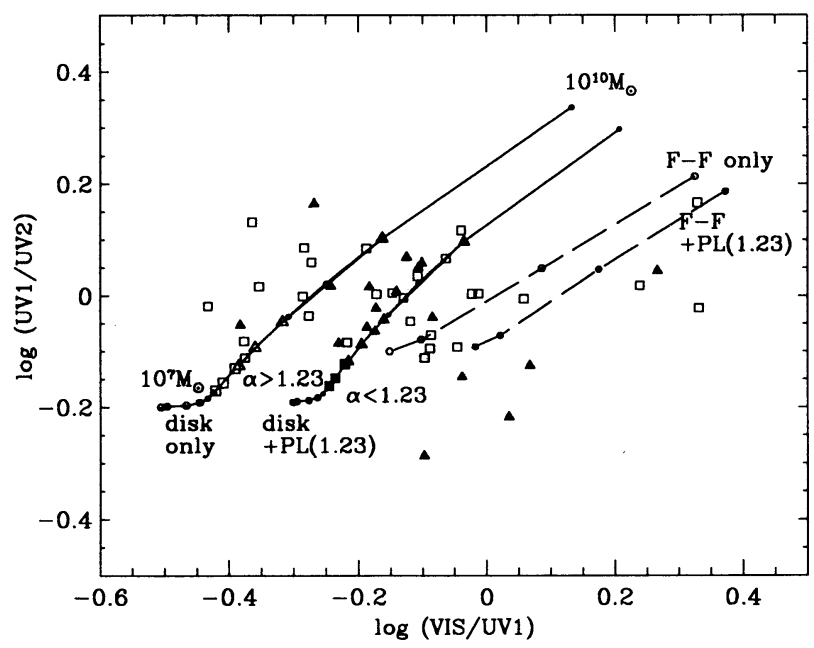

Fig. 14.-VIS/UV1 vs. UV1/UV2 colors for two-component model: accretion disk with nonthermal power-law and free-free model with nonthermal power-law.

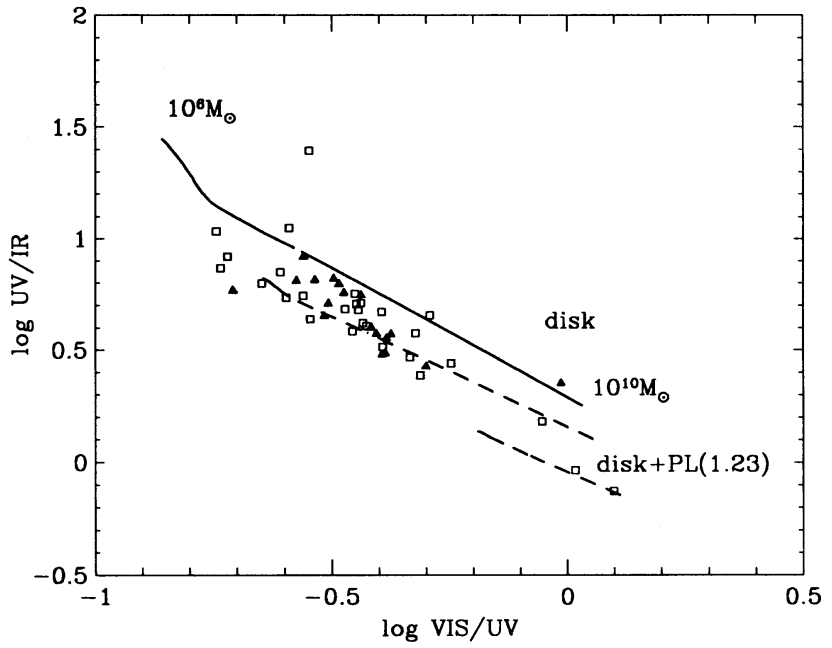

FIG. 15.-VIS/UV vs. UV/IR colors for the accretion disk and with added nonthermal power-law. Power laws have the same spectral index (1.23) but different normalization. Radio-loud quasars are indicated by filled triangles, and radio-quiet quasars are indicated by open squares.

on the assumed power-law slope and normalization. There is a region of allowed power-law slopes for our sample of quasars. The data points are indicated by solid triangles (radio-loud) and empty squares (radio-quiet), and most of them have a $\log (\mathrm{UV} / \mathrm{IR})$ color larger than 0 . There are, however, a few objects with a low UV/IR color $[\log (\mathrm{UV} / \mathrm{IR}) \sim 0]$. These are weak bump quasars (McDowell et al. 1989).

\subsubsection{Hot Dusty Emission}

The contribution from dust becomes important for lower frequencies, $\log v<14.5$. In Figure 16, we plot the IR/VIS/UV colors for the composite spectra where the emission of the optically thick dust is added to the emission from an accretion disk in the Kerr geometry. We plot three curves for the models with the maximum dust temperature of $1750 \mathrm{~K}, 1500 \mathrm{~K}$, and $1100 \mathrm{~K}$. The most data points are located in the region for the

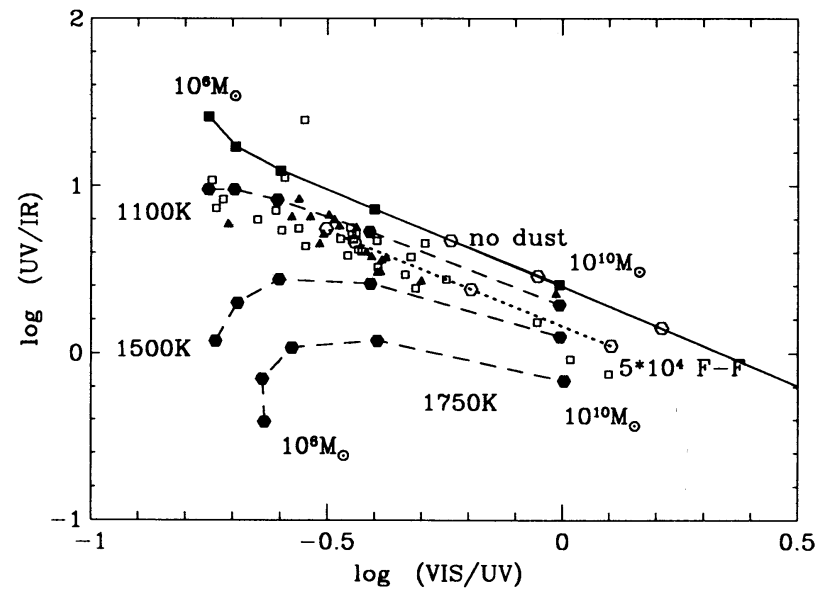

FIG. 16.-VIS/UV vs. UV/IR colors for the accretion disk and accretion disk with the added emission from the hot dust with the different maximum temperatures $1100 \mathrm{~K}, 1500 \mathrm{~K}$, and $1750 \mathrm{~K}$. Dotted line represents the hot dust plus free-free emission for the dust with $1100 \mathrm{~K}$ (the points on the line indicate different free-free temperatures: $5 \times 10^{4} \mathrm{~K}, 10^{5} \mathrm{~K}, 10^{6} \mathrm{~K}$, and $10^{7} \mathrm{~K}$ ). Radioloud quasars are indicated by filled triangles, and radio-quiet quasars are indicated by open squares. 
dust temperature being moderate, $\sim 1100 \mathrm{~K}$. The highest temperature used in our model is definitely too high. The lowest temperature considered, $900 \mathrm{~K}$, does not give much contribution to the IR band. The best temperature range is between $1000-1300 \mathrm{~K}$.

The dotted line in Figure 16 shows modification to the color by hot dust with the temperature of $1100 \mathrm{~K}$ added to the free-free emission. The free-free luminosity is normalized to $10^{43} \mathrm{ergs} \mathrm{s}^{-1}$ in the UV. This normalization constrains a location of the dust inner radius. Modifications to the color are the same as for the hot dust added to the disk emission. With higher dust temperatures, the model line is moving further out of the region, where the most data points are located.

Reradiation of the central continuum by emission from the hot dust can give enough luminosity to account for the infrared luminosity and UV/IR color. There exists, however, a wellknown problem (Sanders et al. 1989) in explaining the $V$-band luminosity when the spectrum is composed only from an accretion disk and hot dust emission, since dust cannot emit significant flux in this band. We illustrate the problem in Figure 12 by the relation between VIS/UV color and the UV band $\left(L_{\mathrm{UV}}\right)$ luminosity. The solid line connecting the filled triangles represents the emission from the accretion disk in Kerr geometry for different central mass $\left(10^{6}-10^{10} M_{\odot}\right)$ and the same accretion rate $\left(\dot{M}=0.3 \dot{M}_{\text {Edd }}\right)$ and inclination angle $(\cos \theta=0.75)$. The dashed line shows the maximum change in the VIS/UV color as a result of a hot dust component with a maximum evaporation temperature of $1750 \mathrm{~K}$. The range of colors reachable with this modfication is small, especially for large central masses. For most objects, there must be a third component added to the disk emission to explain the color.

This result is similar to the result of Sanders et al. (1989). The spectrum in the optical-UV for the large sample of PG quasars was not possible to fit with the two emission components, accretion disk and a hot dust only. They needed one extra component in the optical part to explain the data. Sanders et al. (1989) assumed that the free-free emission from the hot gas originated through the sublimation of a hot dust. If added, this will modify the VIS/UV color in the direction of the data points in Figure 14.

\section{5. $X$-Ray versus Optical-UV Relation}

The question of whether the soft X-ray "excess" below $\sim 0.5$ $\mathrm{keV}$ is the tail of the optical-UV blue bump is still open. The correlation between the soft X-ray slope and the ratio of the UV to $2 \mathrm{keV}$ flux found recently by Walter \& Fink (1993) in the sample of 50 quasars observed by the ROSAT PSPC can be interpreted as evidence for a link between soft X-ray and optical-UV emission. Laor (1994) reported a similar correlation in a sample of 10 optically selected low-redshift quasars. This sample, being optically selected, does not suffer from a potential selection bias in the Walter \& Fink sample (that it is soft X-ray selected and includes only the brightest quasars in the ROSAT All Sky Survey). From other studies (Fiore et al. 1995), the link between optical-UV and soft X-rays is not clearly established.

In this section we use the color-color diagrams to test this link. Figure 17 shows the UV1/UV2 color plotted against the $\mathrm{UV} / \mathrm{SX}$ color for both the model predictions and the Einstein IPC observations of seven objects from our sample (see $\S 3$ ). The range of UV/SX colors available for the disk and free-free models is large, while the spread in the UV1/UV2 color is narrow. It is possible then to constrain more tightly the range of parameters required for the model to explain the UV and

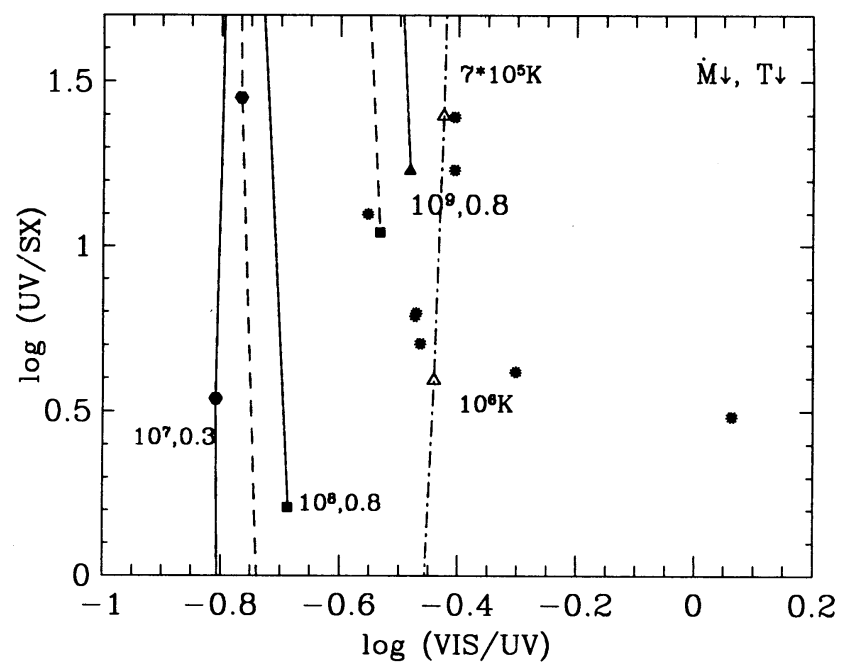

FIG. 17.-UV/SX vs. VIS/UV colors for a set of accretion disk models (modified blackbody): solid line, Kerr; dashed lines, Schwarzschild. The lines connect points with different accretion rates but the same mass and cos $\theta=0.75$. The points indicate parameter values for the models which are located inside the range of plotted colors. The arrows show the direction of the increasing accretion rate or temperature (for free-free models). Dot-dashed line shows the free-free colors. Temperature is indicated by the solid triangle. Stars show the data point of Masnou et al. (1991).

soft-X-ray part of the big blue bump simultaneously. Having the VIS/UV together with the UV/SX allows us to compare the requirements. However, the spread in the VIS/UV is larger than that predicted by models. An extra component (e.g., power-law or irradiation) can increase the value of VIS/UV color. We should note, however, that the observed spread can also be caused artificially by using nonsimultaneous opticalUV data. Also, the IPC "SX" values are not optimal for this kind of study. PSPC data are better (Fiore et al. 1995). The Extreme-Ultraviolet Explorer (EUVE) observations could also be used for further studies.

\section{DISCUSSION}

We have considered how each of the models covers the range of observed colors across the whole of the IR optical UV $\mathrm{X}$-ray part of the spectrum. All the colors and the luminosity are useful in constraining the parameters. We did not consider any far-IR band, mainly because our data are not good enough to study all objects in the sample in this band. Also, we include the dust contribution in a very simple form. Any discussion of the colors of the hot dust in the far-IR requires a more highly developed modeling, which is beyond the scope of this paper.

Here we discuss some general implications and give conclusions for the models.

\subsection{Single-Temperature Bremsstrahlung Emission}

The models do not have the flexibility needed to explain all the observed colors (all the VIS/UV1/UV2 colors fail), even when an extra component (power-law or dust) is added. The shape of the optical-UV spectrum (and colors) is determined by the temperature of the optically thin plasma and is fixed. The range of observed VIS/UV colors is too wide to be explained as a single-temperature thermal bremsstrahlung emission. The uncertainties in the data include variability and it is notable that the observed optical variations are smaller than the UV. This generates a spread of the VIS/UV color for a given source, which is hard to explain in this model. 
We tested a relation between the soft $X$-ray excess and optical-UV band. A very narrow range of model temperatures $\left(7 \times 10^{5} \mathrm{~K}-7 \times 10^{6} \mathrm{~K}\right)$ is required for the observed UV/SX colors to be explained by a single-temperature free-free model. For this temperature region, the UV1/UV2 color range is smaller than the observed range of colors. Fiore et al. (1995) concluded the same for a sample of six objects observed by the ROSAT PSPC. Their sample requires the temperature of the hot plasma to be between $1.5 \times 10^{6} \mathrm{~K}-5 \times 10^{6} \mathrm{~K}$ to fit the observed slopes of soft X-ray excesses. The models that could fit the observed soft X-ray slopes underpredicted the opticalUV luminosities. The temperatures in Fiore et al. are higher than in our case, since ROSAT PSPC data constrain the slope of the soft X-ray excess much better than the Einstein IPC data.

The single-temperature free-free models are not adequate to explain the observed colors for the quasars in our sample. Multitemperature models need to be examined. Models that predict the run of emission measure with temperature are needed to limit the number of free parameters in such models. Accretion disk models provide such a formulation in the optically thick case.

\subsection{Accretion Disks}

The disk models have more flexibility than the singletemperature free-free or blackbody models and the envelope of colors produced by both the Kerr and Schwarzschild models match quite well the observed values (Fig. 7). The spread of colors results from a number of parameters such as mass, accretion rate, inclination angle, and a choice of geometry. However, there are some points outside the model range. An additional component is needed. The simplest set of local blackbody emission disk models cannot explain the dispersion of the VIS/UV, UV1/UV2 colors (Figs. 2-4). Modified blackbody models (Figs. 5-6) provide more dispersion in the same sense as the data but not a sufficient amount.

The full span of observed big blue bump colors is reproduced in models with a large central black hole masses $\left(\gtrsim 10^{9}\right.$ $\left.M_{\odot}\right)$, while only a small area in the color-color diagrams is covered by the models with a small black hole mass $\left(\lesssim 10^{8}\right.$ $\left.M_{\odot}\right)$ (Figs. 3-7).

The color-luminosity diagram gives an extra constraint on model parameters. Local blackbody models work better than modified blackbody models. They can explain all the data points in the UV1/UV2 versus $L(U V 2)$ diagram, while modified blackbody models predict too large a UV1/UV2 color for a few objects in our sample.

Disk emission alone cannot explain both optical and X-ray colors (Fig. 17), but the IPC data contain large uncertainties because of their poor spectral resolution. The UV/SX color demonstrates the diagnostic value of the soft ( $\$ 0.5 \mathrm{keV}) \mathrm{X}$-ray region. Improved spectral resolution is needed to investigate further. ROSAT PSPC spectra may be of some use (Fiore et al. 1994, 1995). For a few objects with exceptionally low foreground obscuration, $E U V E$ spectra (Marshall et al. 1995) will provide a strong discriminant (Puchnarewicz et al. 1995).

\subsection{Irradiation of the Accretion Disk}

We have investigated variations in the colors resulting from the irradiation of the disk by an external source. Such irradiation has been frequently used lately to explain the spectral "humps" seen in X-ray spectra beginning at $\sim 10 \mathrm{keV}$ (George \& Fabian 1991, and references there). We find that irradiation at $\gtrsim 10 \%$ of the disk flux produces major changes in the optical luminosity and therefore in the VIS/UV colors (Figs. 12-13) and mot just in the X-ray region as generally assumed (for example, by Matt et al. 1993). This influence on the opticalUV provides an additional self-consistency test of these models and should be included in all irradiation models for the X-ray spectra in which the optical-UV part has to be emitted by the same cold medium.

Irradiation of the disk surface can explain the observed range of the VIS/UV1 colors for our sample of quasars (Figs. 12-13). For some extreme cases the irradiation flux needs to be large ( $\gtrsim 50 \%$ of the flux generated locally in the disk). However, a few objects ("weak bump" quasars; McDowell et al. 1989) with the largest VIS/UV1 colors still need more contribution in the VIS band than can be given by irradiation.

The critical radius and frequency defined in $\S 4.2 .2$ have interesting observational consequences: the variability of the high-energy radiation will be correlated with the variability of the optical-UV part of the spectrum for low-luminosity objects, while for high-luminosity objects, no correlation will be present.

Effects of irradiation should be considered self-consistently over the whole range of frequencies of the big blue bump.

\subsection{Additional Components}

In the absence of irradiation (or other additional physics), all pure models, both disk and free-free give almost a single line in the IR/VIS/UV color planes. The observed IR/VIS colors thus require an additional component for all models. We tested two possibilities: hot dust emission and nonthermal power-law components. We can constrain the evaporation temperature of the hot dust for our sample of objects to $900-1500 \mathrm{~K}$. The whole range of observable VIS/UV colors can be achieved with the sum of any pure disk or free-free model and a nonthermal power-law. However, this nonthermal power-law has no strong physical meaning.

\subsection{Outlier Quasars}

The color-color and color-luminosity diagrams allow us also to identify unusual objects. The majority of quasars from our sample are located in small regions in the color-color diagrams, but there are a few objects which are outliers from these "common" colors: for example, "weak bump" quasars or quasars with UV1/UV2 $>0$, which indicates a turnover in their spectra. These outliers are of extreme importance for models, since their colors require extreme sets of parameters to explain them. If the turnover is intrinsic to the primary emission component, then the maximum temperature of the thermal component may be calculated. When it is caused by absorption (internal to the quasar or extragalactic), then studies over a broad range of frequencies are needed in order to understand the characteristics of the absorber.

\section{CONCLUSIONS}

From the present study we can make the following conclusions.

1. Modified blackbody accretion disk models can reproduce both the luminosities and the colors of the quasars in our sample, except for soft X-rays. However, to do this requires that the Kerr geometry applies for some objects, while the Schwarzschild geometry applies for others. Either geometry alone needs an additional component to fit $\sim 25 \%$ of the sample. PSPC and $E U V E$ spectra should help in testing the models for the optical-X-ray connection. 
2. An additional power law helps to explain the optical-UV color for disk models, but it is not needed when the irradiation of the disk is included in the model.

3. Even modest $(10 \%)$ irradiation is an important contribution to the disk models. Including irradiation in the disk may provide a good model for the optical UV X-ray spectra. A self-consistent treatment in optical and X-rays is needed when irradiation is used to explain "Compton humps" in X-ray spectra.

4. The simplest, single-temperature, free-free models need additional components or a range of temperatures to cover the observed colors. A prescription for emission measure versus temperature is needed.

5. The best range of the optically thick hot dust temperature is between $900-1500 \mathrm{~K}$, and $1750 \mathrm{~K}$ is definitely too high. More detailed modeling of the reddening caused by the opti- cally thin shell of hot dust is needed to estimate selfconsistently its effect on the IR optical UV colors.

6. The reality of the spread in colors is crucial to modeling. Thus, outlier quasars in the color-color diagrams are of great importance. Detailed follow-up observations of both "weak bump" and "UV-turnover" candidates are needed.

We thank Ari Laor for kindly providing us with his transfer function code for the Kerr geometry. The numerical code to calculate disk spectra was developed from the original version provided by Bożena Czerny. We also thank the referee, Jill Bechtold, for her comments which improved the paper. This work was supported by NASA grants NAGW-2201 (LTSA), NAG 5-1872, NAG 5-1883, and NAG 5-1536 (ROSAT), and NASA contracts NAS 5-30934 (RSDC), NAS 5-30751 (HEAO 2) and NAS 8-39073 (ASC).

\section{APPENDIX}

\section{LUMINOSITIES IN THE COLOR BANDS}

Table 3 contains accretion disk models in a Kerr geometry with local blackbody emission, Table 4 is similar, but for modified blackbody emission. Table 5 contains accretion disk models in a Schwarzschild geometry with modified blackbody emission. Table 6 shows the free-free model.

TABLE 3

ACCRETION Disk MODELS (KeRR, LOCAL BLACKBODY) ${ }^{a}$

\begin{tabular}{ccccccc}
\hline \hline Mass $\left(M_{\odot}\right)$ & $\dot{M} / \dot{M}_{\text {Edd }}$ & $(0.8-1.6 \mu \mathrm{m})$ & $(4000-8000 \AA)$ & $(1400-2000 \AA)$ & $(1000-1400 \AA)$ & $(1000-2000 \AA)$ \\
\hline $10^{6} \ldots \ldots \ldots$ & 0.1 & 40.62 & 41.10 & 41.54 & 41.73 & 41.89 \\
& 0.3 & 40.87 & 41.40 & 41.86 & 42.06 & 42.22 \\
$10^{7} \ldots \ldots \ldots$ & 0.8 & 41.08 & 41.65 & 42.15 & 42.35 & 42.50 \\
& 0.3 & 42.02 & 42.43 & 42.86 & 43.03 & 43.19 \\
$10^{8} \ldots \ldots \ldots$ & 0.8 & 42.32 & 42.75 & 43.19 & 43.37 & 43.53 \\
& 0.3 & 42.57 & 43.03 & 43.48 & 43.67 & 43.82 \\
$10^{9} \ldots \ldots \ldots$ & 0.8 & 43.37 & 43.76 & 44.15 & 44.28 & 44.46 \\
& 0.3 & 43.68 & 44.08 & 44.49 & 44.65 & 44.82 \\
$10^{10} \ldots \ldots .$. & 0.8 & 44.69 & 44.37 & 44.79 & 44.96 & 45.12 \\
& 0.01 & 45.02 & 45.407 & 45.39 & 45.44 & 45.65 \\
& 0.1 & 45.30 & 45.69 & 45.76 & 45.85 & 46.05 \\
& 0.3 & 46.01 & 45.59 & 46.08 & 46.20 & 46.39 \\
& 0.8 & 46.34 & 46.36 & 46.52 & 45.12 & 45.56 \\
\end{tabular}

Note.-Table 3 is published in its entirety in computer readable form in the AAS CD-ROM Series, Vol. 5.

${ }^{a} \cos \theta=0.75$ for all the models. Values are logarithms of luminosity in units of ergs $\mathrm{s}^{-1}$.

TABLE 4

ACCretion Disk Models (KerR, Modified BlaCkbOdy) ${ }^{\mathrm{a}}$

\begin{tabular}{ccccccc}
\hline \hline Mass $\left(M_{\odot}\right)$ & $\dot{M} / \dot{M}_{\text {Edd }}$ & $(0.8-1.6 \mu \mathrm{m})$ & $(4000-8000 \AA)$ & $(1400-2000 \AA)$ & $(1000-1400 \AA)$ & $(1000-2000 \AA)$ \\
\hline $10^{7} \ldots \ldots \ldots$ & 0.1 & 41.95 & 42.44 & 42.89 & 43.08 & 43.23 \\
& 0.3 & 42.20 & 42.74 & 43.22 & 43.88 & 43.55 \\
$10^{8} \ldots \ldots \ldots$ & 0.1 & 42.40 & 43.00 & 43.50 & 43.64 & 43.82 \\
& 0.3 & 43.36 & 43.78 & 44.19 & 44.32 & 44.50 \\
$10^{9} \ldots \ldots \ldots$ & 0.8 & 43.96 & 44.10 & 44.51 & 44.60 & 44.80 \\
& 0.1 & 44.71 & 44.38 & 44.76 & 44.81 & 45.03 \\
$10^{10} \ldots \ldots .$. & 0.8 & 45.03 & 45.10 & 45.43 & 45.44 & 45.67 \\
& 0.1 & 45.30 & 45.43 & 45.72 & 45.72 & 45.95 \\
& 0.3 & 46.03 & 46.70 & 45.92 & 45.89 & 46.14 \\
& 0.8 & 46.63 & 46.38 & 46.52 & 46.39 & 46.70 \\
\end{tabular}

NoTE.-Table 4 is published in its entirety in computer readable form in the AAS CD-ROM Series, Vol. 5.

${ }^{a} \cos \theta=0.75$ for all the models. Values are logarithms of luminosity in units of ergs $\mathrm{s}^{-1}$. 
TABLE 5

ACCRETION Disk Models (SchWARZSChild, Modified BlaCKBody) ${ }^{\mathrm{a}}$

\begin{tabular}{ccccccc}
\hline Mass $\left(M_{\odot}\right)$ & $\dot{M} / \dot{M}_{\text {Edd }}$ & $(0.8-1.6 \mu \mathrm{m})$ & $(4000-8000 \AA)$ & $(1400-2000 \AA)$ & $(1000-1400 \AA)$ & $(1000-2000 \AA)$ \\
\hline $10^{7} \ldots \ldots \ldots$ & 0.1 & 41.89 & 42.35 & 42.67 & 42.74 & 42.95 \\
& 0.3 & 42.15 & 42.67 & 43.05 & 43.16 & 43.35 \\
$10^{8} \ldots \ldots \ldots$ & 0.1 & 42.36 & 42.93 & 43.39 & 43.53 & 43.71 \\
& 0.3 & 43.59 & 43.64 & 43.83 & 43.76 & 44.03 \\
$10^{9} \ldots \ldots \ldots$ & 0.8 & 43.84 & 43.99 & 44.26 & 44.26 & 44.50 \\
& 0.1 & 44.59 & 44.29 & 44.63 & 44.68 & 44.89 \\
$10^{10} \ldots \ldots \ldots$ & 0.3 & 44.93 & 45.25 & 44.80 & 44.49 & 44.91 \\
& 0.8 & 45.22 & 45.58 & 45.33 & 45.14 & 45.48 \\
& 0.3 & 45.83 & 46.02 & 45.76 & 45.64 & 45.94 \\
& 0.8 & 46.20 & 46.44 & 46.14 & 44.80 & 45.67 \\
\hline
\end{tabular}

NoTE.-Table 5 is published in its entirety in computer-readable form in the AAS CD-ROM Series, Vol. 5.

${ }^{a} \cos \theta=0.75$ for all the models. Values are logarithms of luminosity in units of ergs $\mathrm{s}^{-1}$.

TABLE 6

FreE-FreE MODEL ${ }^{\text {a }}$

\begin{tabular}{cccccc}
\hline \hline$T(\mathrm{~K})$ & $(0.8-1.6 \mu \mathrm{m})$ & $(4000-8000 \AA)$ & $(1400-2000 \AA)$ & $(1000-1400 \AA)$ & $(1000-2000 \AA)$ \\
\hline $5 \times 10^{4} \ldots \ldots$ & 42.85 & 43.07 & 42.74 & 42.53 & 42.96 \\
$10^{5} \ldots \ldots \ldots \ldots$ & 42.72 & 42.99 & 42.90 & 42.85 & 43.18 \\
$10^{6} \ldots \ldots \ldots \ldots$ & 42.61 & 42.93 & 43.03 & 43.11 & 43.37 \\
$10^{7} \ldots \ldots \ldots \ldots$ & 42.56 & 42.91 & 43.06 & 43.16 & 43.41 \\
\hline
\end{tabular}

NoTE.-Table 6 is published in its entirety in computer-readable form in the AAS CD-ROM Series. Vol. 5.

a Values are logarithms of luminosity in units of ergs $\mathbf{s}^{-1}$. Model luminosities are normalized to the average luminosity of our sample at $\log v=14.5$.

\section{REFERENCES}

Antonucci, R. 1994, in IAU Symp. 159, Multi-wavelength Continuum Emission of AGN, Proc. ed. T. J.-L. Courvoisier \& A. Blecha (Dordrecht: Kluwer), 301

Barvainis, R. 1990, ApJ, 353, 419

1993, ApJ, 412, 513

Bechtold, J., Czerny, B., Elvis, M., Fabbiano, G., \& Green, R. F. 1987, ApJ, 314, 699

Bechtold, J., Elvis, M., Fiore, F., Kuhn, O., Cutri, R. M., McDowell, J. C.,

Rieke, M., Siemiginowska, A., \& Wilkes, B. J. 1994, AJ, 108, 374

Boyle, B., et al. 1990, MNRAS, 243, 1

Brissenden, R. J. V. 1989, Ph.D. thesis, Australian National University

Caditz, D. 1993, ApJ, 404, 539

Carleton, N. P., Elvis, M., Fabbiano, G., Willner, S. P., Lawrence, A., \& Ward, M. 1987, ApJ, 318, 595

Clavel, J., et al. 1992, ApJ, 393, 113

Collin-Souffrin, S., Czerny, B., Dumont, A.-M., \& Życki, P. T. 1995, A\&A, submitted

Cunningham, C. T. 1975, ApJ, 202, 788

.1976, ApJ, 208, 534

Cutri, R. M., Wiśniewski, W. Z., Rieke, G. H., \& Lebofsky, M. J. 1985, ApJ, 296,423

Czerny, B. 1994, in IAU Symp. 159, Multi-wavelength Continuum Emission of AGN, ed. T. J.-L. Courvoisier \& A. Blecha (Dordrecht: Kluwer), 261

Czerny, B., Czerny, M., \& Grindlay, J. E. 1986, ApJ, 311, 241

Czerny, B., \& Elvis, M. 1987, ApJ, 321, 305

Czerny, B., Jaroszyński, M., \& Czerny, M. 1994, MNRAS, 268, 135

Elvis, M., Green, R. F., Bechtold, J., Schmidt, M., Neugebauer, B. T., Soifer B. T., Matthews, K., \& Fabbiano, G. 1986, ApJ, 310, 291

Elvis, M., \& Lawrence, A. 1985, in Astrophysics of Active Galaxies, ed. J. S. Miller (Mill Valley, CA: University Science Books), 289

Elvis, M., Wilkes, B. J., McDowell, J. C., Green, R. F., Bechtold, J., Willner, S. P., Polomski, E., \& Cutri, R. 1994, ApJS, 95, 1 (Paper I)

Fiore, F., Elvis, E., McDowell, J. C., Siemiginowska, A., \& Wilkes, B. J. 1994, ApJ, 431, 515

Fiore, F., Elvis, E., Siemiginowska, A., Wilkes, B. J., McDowell, J. C., \& Mathur, S. 1995, ApJ, 449, 74

Frank, J., King, A. R., \& Raine, D. J. 1992, Accretion Power in Astrophysics (Cambridge: Cambridge Univ. Press)

George, I. M., \& Fabian, A. C. 1991, MNRAS, 249, 352

Glass, I. S. 1992, MNRAS, 256, 23P

Gronenschild, E. H. B. M., \& Mewe, R. 1978, A\&AS, 32, 283

Grossan, B. A. 1993, Ph.D. thesis, MIT
Guilbert, P. W., \& Rees, M. J. 1988, MNRAS, 233, 475

Hertzsprung, E. 1905, Zur Strahlung der Sterne (Giants and Dwarfs). Z. Wiss Photog. 3, english translation (1960) in Source Book in Astronomy, ed. H. Shapley (Cambridge: Harvard University Press)

Kinney, A. L., Bohlin, R. C., Blades, J. C., \& York, D. G. 1991, ApJS, 75, 645

Ko, Y.-K., \& Kallman, T. R. 1991, ApJ, 374, 721

Kuhn, O., Bechtold, J., Cutri, R., Elvis, M., \& Rieke, M. 1995, ApJ, 438, 643

Laor, A. 1994, ApJ, 435, 611

Laor, A. 1990, MNRAS, 246, 396

Laor, A., \& Draine, B. T. 1993, ApJ, 402, 441

Laor, A., \& Netzer, H. 1989, MNRAS, 238, 897

Laor, A., Netzer, H., \& Piran, T. 1990, MNRAS, 242, 560

Loska, Z., Szczerba, R., \& Czerny, B. 1993, MNRAS, 261, 63

Malkan, M. A. 1983, ApJ, 268, 582

1991, in Structure and Emission Properties of Accretion Disks, ed.

C. Bertout, S. Collin, J.-P. Lasota, \& J. Tran Than Van (Gif-sur-Yvette Cedex: Editions Frontières), 165

Maraschi, L., \& Molendi, S. 1990, ApJ, 353, 452

Marshall, H., et al. 1995, ApJ, 439, 90

Masnou, J.-L., Wilkes, B. J., Elvis, M., McDowell, J. C., \& Arnaud, K. A. 1992, A\&A, 253, 35

Matt, G., Fabian, A. C., \& Ross, R. R. 1993, MNRAS, 264, 839

McDowell, J. C., Elvis, M., Wilkes, B. J., Willner, S. P., Oey, M. S., Polomski, E., Bechtold, J., \& Green, R. F. 1989, ApJ, 354, L19

Mushotsky, R. F., Done, C., \& Pounds, K. A. 1993, ARA\&A, 31, 717

Nandra, K., \& George, I. M. 1994, MNRAS, 267, 974

Novikov, I. D., \& Thorne, K. S. 1973, in Black Holes, ed. C. DeWitt \& B. DeWitt (New York: Gordon \& Breach), 344

Page, D. N., \& Thorne, K. S. 1974, ApJ, 191, 499

Puchnarewicz, E., Mason, K. O., Siemiginowska, A., \& Pounds, K. 1995,

MNRAS, in press

Ross, R. R., \& Fabian, A. C. 1993, MNRAS, 261, 74

Russell, H. N. 1912, Proc. Am. Phil. Soc., 51, 569

1913, Science, 37, 651

Rybicki, G. B., \& Lightman, A. B. 1979, Radiative Processes in Astrophysics

(New York: Wiley)

Sandage, A. 1957, ApJ, 125, 422

. 1973, ApJ, 180, 687

Sanders, D. B., Phinney, E. S., Neugebauer, G., Soifer, B. T., \& Matthews, K. 1989, ApJ, 347, 29

Schmidt, M., \& Green, R. F. 1983, ApJ, 269, 352

Shakura, N. I., \& Sunyaev, R. A. 1973, A\&A, 24, 337 
SIEMIGINOWSKA ET AL.

Van der Klis, M. 1989, ARA\&A, 27

Shields, G. A. 1979, Nature, 272, 706

Sitko, M., Sitko, A. K., Siemiginowska, A., \& Szczerba, R. 1993, ApJ, 409, 139

Störzer, H. 1993, A\&A, 271, 25

Sun, W.-H., \& Malkan, M. A. 1989, ApJ, 346, 68

Svensson, R. 1984, MNRAS, 209, 175

InI Tinsley, B. A. 1973, ApJ, 186, 35

III Turner, J. L., \& Pounds, K. A. 1989, MNRAS, 240, 833

Tripp, T. M., Bechtold, J., \& Green, R. F. 1994, ApJ, 433, 533

Véron-Cetty, M. P., \& Véron, P., A Catalogue of Quasars and Active Galaxies, (4th ed.; ESO Sci. Rep. No. 7)

Walter, R., \& Fink, H. H. 1993, A\&A, 274, 105

Wandel, A. 1991, A\&A, 241, 5

Wandel, A., \& Petrosian, V. 1988, ApJ, 329, L11

Wilkes, B. J., \& Elvis, M. 1987, ApJ, 323, 243

Wills, B. J., Netzer, H., \& Wills, D. 1985, ApJ, 188, 94 